\title{
Investigation of microbial community interactions between lake Washington methanotrophs using genome-scale metabolic modeling
}

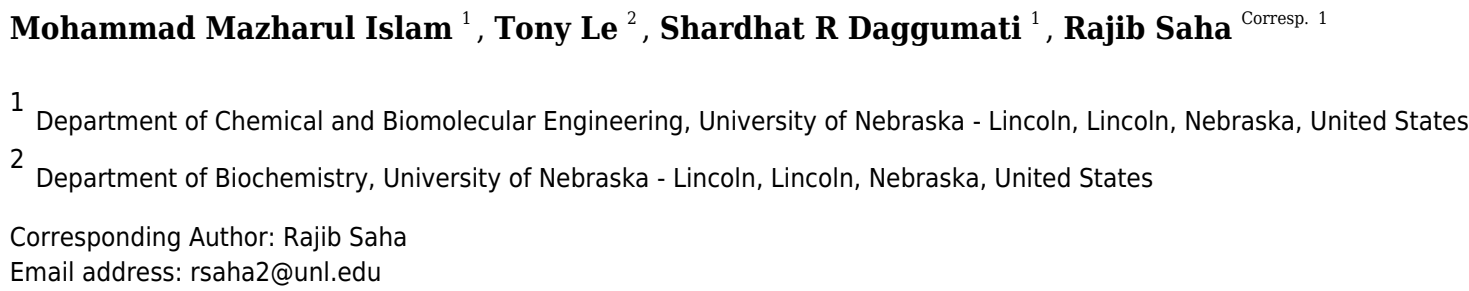

Abstract Background. The role of methane in global warming has become paramount to the environment and the human society, especially in the past few decades. Methane cycling microbial communities play an important role in the global methane cycle, which is why the characterization of these communities is critical to understand and manipulate their behavior. Methanotrophs are a major player in these communities and are able to oxidize methane as their primary carbon source. Results. Lake Washington is a freshwater lake characterized by a methane-oxygen countergradient that contains a methane cycling microbial community. Methanotrophs are a major part of this community involved in assimilating methane from lake water. Two significant methanotrophic species in this community are Methylobacter and Methylomonas. In this work, these methanotrophs are computationally studied via developing highly curated genome-scale metabolic models. Each model was then integrated to form a community model with a multi-level optimization framework. The competitive and mutualistic metabolic interactions among Methylobacter and Methylomonas were also characterized. The community model was next tested under carbon, oxygen, and nitrogen limited conditions in addition to a nutrient-rich condition to observe the systematic shifts in the internal metabolic pathways and extracellular metabolite exchanges. Each condition showed variations in the methane oxidation pathway, pyruvate metabolism, and the TCA cycle as well as the excretion of formaldehyde and carbon di-oxide in the community. Finally, the community model was simulated under fixed ratios of these two members to reflect the opposing behavior in the two-member synthetic community and in sediment-incubated communities. The community simulations predicted a noticeable switch in intracellular carbon metabolism and formaldehyde transfer between community members in sediment-incubated vs. synthetic condition. Conclusion. In this work, we attempted to predict the response of a simplified methane cycling microbial community from Lake Washington to varying 
environments and also provide an insight into the difference of dynamics in sedimentincubated microcosm community and synthetic co-cultures. Overall, this study lays the ground for in silico systems-level studies of freshwater lake ecosystems, which can drive future efforts of understanding, engineering, and modifying these communities for dealing with global warming issues. 
1 Investigation of microbial community interactions between lake 2 Washington methanotrophs using genome-scale metabolic modeling

3

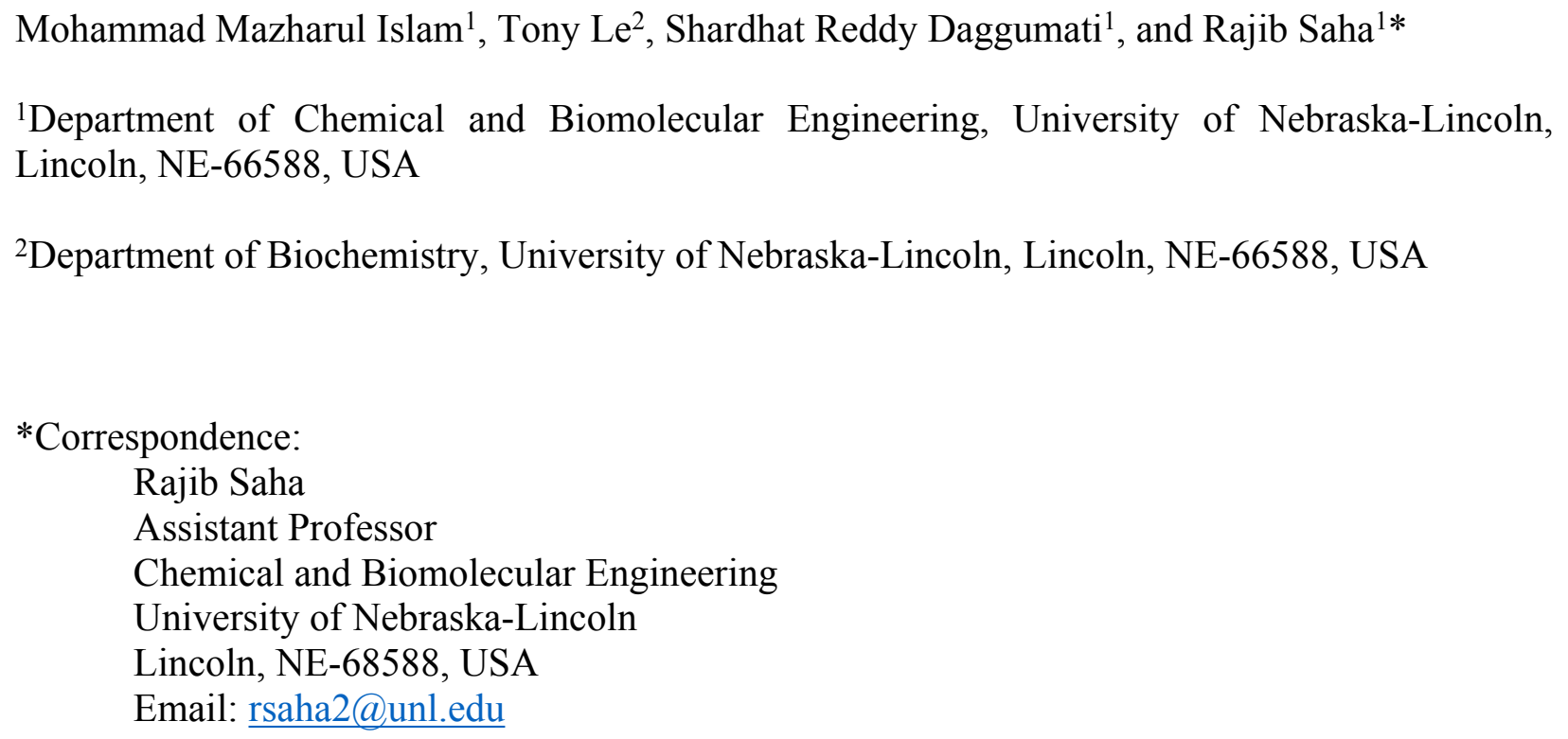

Keywords: Lake Washington, Methylobacter tundripaludum 21/22, Methylomonas sp. LW13, microbial community, genome-scale metabolic modeling, methane-cycling community 


\section{Abstract}

28 Background. The role of methane in global warming has become paramount to the environment and the human society, especially in the past few decades. Methane cycling microbial communities play an important role in the global methane cycle, which is why the characterization of these communities is critical to understand and manipulate their behavior. Methanotrophs are a major player in these communities and are able to oxidize methane as their primary carbon source.

Results. Lake Washington is a freshwater lake characterized by a methane-oxygen countergradient that contains a methane cycling microbial community. Methanotrophs are a major part of this community involved in assimilating methane from lake water. Two significant methanotrophic species in this community are Methylobacter and Methylomonas. In this work, these methanotrophs are computationally studied via developing highly curated genome-scale metabolic models. Each model was then integrated to form a community model with a multi-level optimization framework. The competitive and mutualistic metabolic interactions among Methylobacter and Methylomonas were also characterized. The community model was next tested under carbon, oxygen, and nitrogen limited conditions in addition to a nutrient-rich condition to observe the systematic shifts in the internal metabolic pathways and extracellular metabolite exchanges. Each condition showed variations in the methane oxidation pathway, pyruvate metabolism, and the TCA cycle as well as the excretion of formaldehyde and carbon di-oxide in the community. Finally, the community model was simulated under fixed ratios of these two members to reflect the opposing behavior in the two-member synthetic community and in sediment-incubated communities. The community simulations predicted a noticeable switch in intracellular carbon metabolism and formaldehyde transfer between community members in sediment-incubated vs. synthetic condition.

Conclusion. In this work, we attempted to predict the response of a simplified methane cycling microbial community from Lake Washington to varying environments and also provide an insight into the difference of dynamics in sediment-incubated microcosm community and synthetic cocultures. Overall, this study lays the ground for in silico systems-level studies of freshwater lake ecosystems, which can drive future efforts of understanding, engineering, and modifying these communities for dealing with global warming issues. 
56

57

58

59

60

61

62

63

64

65

66

67

68

69

70

71

72

73

74

75

76

77

78

79

80

81

82

83

84

85

86

87

88

89

90

91

92

93

94

95

96

97

98

99

100

101

102

\section{Introduction}

The accelerated rise in worldwide average temperature in recent years is posing a serious threat to the environment, terrestrial ecosystems, human health, economy, and the ultimate survival of the planet earth. About 20 percent of global warming is caused by methane and it is expected to be 86 times more potent than carbon di-oxide in warming the earth over the next two decades (Houghton, Jenkins \& Ephraums, 1990; IPCC, 2013). The impacts of the rapid increase in atmospheric methane (Nisbet et al., 2019) are compounded as higher temperatures are associated with an increase in methane production from wetlands and lakes (Yvon-Durocher et al., 2014). Aerobic methanotrophs are mostly gram-negative Proteobacteria that are an integral part of the global carbon cycle (Hanson \& Hanson, 1996). They exist in diverse environments such as wetlands, lakes, and the tundra and use the enzyme methane monooxygenase (MMO) to oxidize methane as their sole source of carbon (Hanson \& Hanson, 1996). Methane-oxidizing Verrucomicrobia found in geothermal acidic environments are also involved in methane oxidation while solely using carbon dioxide in the Calvin cycle (Carere et al. 2019; Mohammadi et al. 2019; van Teeseling et al. 2014). The anaerobic methanotrophic archaea are also an important sink of methane that couple methane oxidation with sulfate reduction mediated by sulfate-reducing bacteria (Cui et al. 2015). Thus, methanotrophs act as the primary biological sink for methane (Hanson \& Hanson, 1996), consuming up to 90 percent of the methane produced in soil/sediments in addition to the atmospheric methane (Whalen \& Reeburgh, 1990; Krause et al., 2017). Methanotrophs have also shown the ability to produce various useful products such as single-cell proteins, biodiesels, biopolymers, and osmo-protectants (Strong, Xie \& Clarke, 2015).

Lakes act as major sources and sinks for methane and account for 6 to 16 percent of biologically produced methane (IPCC, 2013; Yvon-Durocher et al., 2014). Lake Washington is a freshwater lake characterized by a methane-cycling community where methanotrophs are one of the important functional microbial groups involved in methane oxidation (Yu et al., 2016). It contains a steep vertical counter-gradient of methane and oxygen, and is separated into oxic and anoxic layers where methane production and consumption occur, respectively (Yu et al., 2016). Hence, it can be a model system to better understand methane-cycling communities in lakes and their role in the global methane cycle. Understanding the metabolic interactions in these communities will aid in developing methods to reduce the amount of methane emitted from lakes. A diverse array of microbes exist in the Lake Washington community, where Proteobacteria comprise 33\% of the community and includes a major subtype of methanotrophs, Methylococcaceae at a 10\% abundance level (Beck et al., 2013). The genus Methylobacter is the most dominant player in Methylococcaceae group at 47.7\%, while other major players include Crenothrix, Methylomonas, and Methylomicrobium at 30.0\%, 10.8\%, and 7.4\%, respectively (Beck et al., 2013). Other members include cyanobacteria, bacteriodetes, acidobacteria, and chloroflexi (Beck et al., 2013).

Understanding the physiological dynamics and interactions in natural methane-cycling communities, such as the Lake Washington, are crucial to addressing problems concerning methane's role in global warming and leveraging methanotrophs' possible functions in bioremediation and bioproduction. Omics-based techniques and high-throughput sequencing can elucidate important features of the community such as taxonomic information, community composition, and presence of functionally important genes (Temperton \& Giovannoni, 2012). However, it is difficult to assign functionality to members of the community and decipher the roles of individual players due to the complexity of the community and the data involved (Zengler, 2009; Zengler \& Palsson, 2012). On the other hand, synthetic communities were proven to be efficient

Peer] reviewing PDF | (2020:02:45973:2:0:NEW 28 May 2020) 
models to provide insight into metabolic capabilities and interactions (De Roy et al., 2014). Simple representative community structures can be made to lower complexity, achieve more consistent results, and efficiently elucidate inter-species interactions (De Roy et al., 2014). Certain Lake Washington community members are easier to cultivate in a laboratory setting than other members. For instance, Methylomonas and Methylosinus species previously shown ease of cultivation in the laboratory (Auman et al., 2000). However, Methylobacter species were difficult to isolate and demonstrated poor growth compared to Methylomonas and Methylosinus (Yu et al., 2016). A synthetic community comprising 50 different Lake Washington microbes belonging to 10 methanotrophic, 36 methylotrophic, and 4 non-methanotrophic heterobacteria showed that Methylobacter was outperformed by Methylomonas in the community (Yu et al., 2016). Similar results about the dominance of Methylomonas in pure cultures and in standard conditions were observed in their later experiments (Yu et al. 2017). These observations were inconsistent with previous stable isotope probing studies that found Methylobacter is the dominant Methylococcaceae species among microbes from Lake Washington when grown on methane (Beck et al. 2013; Kalyuzhnaya et al. 2008). These inconsistencies indicate that the complexities of biological systems often make it challenging to understand the functions and interactions within and among organisms in synthetic communities via in vitro and in vivo studies.

In silico evaluation and analysis utilizing mathematical relation-based modeling allow for a highresolution understanding of the biological processes in a microbial community. The availability of genome-scale metabolic network models combined with biological constraints provide multiple methods to analyze, perform in silico experiment, develop hypotheses, and redesign biological systems at a genome-level (Zomorrodi \& Maranas, 2012; Zomorrodi, Islam \& Maranas, 2014; Maranas \& Zomorrodi, 2016; Islam \& Saha, 2018; (Alsiyabi et al. 2019; Islam et al. 2020; Schroeder et al. 2020; Schroeder \& Saha 2019). To develop effective multi-species community models, significant and comprehensive knowledge of inter-species interactions and experimental data must be utilized. Compartmentalized community metabolic models were used to model simple microbial consortia involved in bioremediation, synthetic auxotrophic co-growth, human gut microbiome, and soil bacterial ecosystems (Stolyar et al., 2007; Bizukojc et al., 2010; Lewis et al., 2010; Zhuang et al., 2011; Shoaie \& Nielsen, 2014; Henry et al., 2016). On the other hand, community modeling frameworks incorporating the trade-offs between species- and communitylevel fitness successfully modeled steady-state and dynamic behavior in naturally occurring and synthetic soil microbial communities, synthetic co-cultures for bioproduction, human gut microbiome, and very recently to understand the microbial interactions in bovine rumen and viral influences (Zomorrodi \& Maranas, 2012; Zomorrodi, Islam \& Maranas, 2014; Chan, Simons \& Maranas, 2017; Islam et al., 2019). Other novel methods were also proposed for modeling of such communities involving elementary mode analysis, evolutionary game theory, nonlinear dynamics, and stochastic processes (Vallino, 2003; Lehmann \& Keller, 2006; Shou, Ram \& Vilar, 2007; Borenstein \& Feldman, 2009; Freilich et al., 2009; Frey, 2010; Nadell, Foster \& Xavier, 2010; Magnúsdóttir, Heinken \& Kutt, 2017). While there have been multiple studies in recent years involved in the model development of various methanotrophs (Akberdin et al. 2018; Bordel et al. 2019a; Bordel et al. 2019b; de la Torre et al. 2015; Lieven et al. 2018; Naizabekov \& Lee 2020), an integrated community level analysis of freshwater methane utilizing ecosystems have not been performed yet. 
148 In this work, we developed a simplified community metabolic model with two representative and functionally important strains of Lake Washington, namely, Methylobacter tundripaludum 21/22 (hereafter, Methylobacter) and Methylomonas sp. LW13 (hereafter, Methylomonas) as representative organisms of the methane-oxidizing microbes in the Lake Washington ecosystem. These species were chosen because of their availability in Lake Washington sediments, the ability to mitigate common pollutants, and produce desirable biological products, and the availability of genome-annotation. Draft models of these species were reconstructed followed by careful curation to ensure proper representation of the species. Metabolic pathways and individual reactions that are fundamental to the growth of these organisms such as the ribulose monophosphate pathway, pentose phosphate pathway, methane metabolism, amino acid synthesis and utilization, serine cycle, and Coenzyme B12 biosynthesis were manually scrutinized and then integrated into the models. The metabolites exchanged by the two species models were established by referring to literature and known transporter information (Boden et al. 2011; Caspi et al. 2016; Elbourne et al. 2017; Henry et al. 2010; Kanehisa 2008; Nguyen et al. 2018; Orata et al. 2018; Svenning et al. 2011; Szklarczyk et al. 2017; UniProt Consortium 2018; Wartiainen et al. 2006). The curated models of Methylobacter (704 genes, 1329 metabolites, and 1404 reactions) and Methylomonas (658 genes, 1378 metabolites, and 1391 reactions) were then utilized to develop a community model using a multi-level optimization framework, which was used to estimate biologically feasible metabolite secretion profiles and community compositions (Zomorrodi \& Maranas, 2012; Zomorrodi, Islam \& Maranas, 2014; Islam et al., 2019). The community was placed under carbon, oxygen, and nitrogen-limiting as well as nutrient-rich environments to study the changes in intracellular carbon and nitrogen metabolism and metabolite excretion profiles. The community composition of carbon-limited environments predicted a shift in the carbon metabolism of both species. The community also demonstrates conservative metabolism under oxygen and carbonlimited environments and produce less carbon-di-oxide. Under these conditions, the mutualistic behavior involving formaldehyde transfer between Methylobacter and Methylomonas is rarely observed. Our results also indicate metabolic reprogramming in TCA cycle and pyruvate metabolism, which can help generate new hypotheses for in vivo experiments. We also simulated the observed binary compositions in a sediment-incubated community and a synthetic co-culture to predict the changes in intra- and extracellular metabolic fluxes. Overall, our results enhance the mechanistic understanding of the Lake Washington methane-cycling community, which can drive further engineering efforts for efficient rerouting of carbon and nitrogen as well as mitigation of methane emission from freshwater ecosystems globally.

\section{Materials \& Methods}

\section{Metabolic model reconstruction}

The draft genome-scale metabolic models of Methylobacter and Methylomonas were developed and downloaded using the ModelSEED database (Henry et al., 2010). The models included reactions for glycolysis/gluconeogenesis, citrate cycle, pentose phosphate pathway, steroid biosynthesis, nucleotide metabolism, and various amino acid biosynthesis. Flux Balance Analysis (FBA), a mathematical approach for analyzing the flow of metabolites through a metabolic 
190

network, was utilized for model testing and analyzing flux distributions throughout the work (Orth, Thiele \& Palsson, 2010). FBA implements the following optimization framework.

$$
\operatorname{Maximize}_{\left(v_{j}\right)} \quad v_{\text {biomass }}
$$

subject to

$$
\sum_{j \in J} S_{i j} \cdot v_{j}=0 \quad \forall i \in I
$$

$$
L B_{j} \leq v_{j} \leq U B_{j} \quad \forall j \in I
$$

In the framework, $I$ and $J$ represent the sets of metabolites and reactions in the metabolic model, respectively. $S_{i j}$ represents the stoichiometric coefficient of metabolite $i$ in reaction $j$. The flux value of each reaction $j, v_{j}$, must be within the parameters of the minimum, $L B_{j}$, and maximum, $U B_{j}$, biologically allowable fluxes. $v_{\text {biomass }}$ is the flux of the biomass reaction which simulates the yield of cellular growth in the model (Orth, Thiele \& Palsson, 2010).

The biomass composition from recently published methanotroph model Methylomicrobium buryatense strain 5G(B1)(de la Torre et al. 2015) was adopted in this study with slight modification in the lipid macromolecular stoichiometry to account for different lipid macromolecules in the models (see Supplementary Data S1). Non-growth associated ATP maintenance flux was set to $21.6 \mathrm{mmol} / \mathrm{gDCW} . \mathrm{hr}$ according to calculated values in the closely related organism Methylomicrobium alcaliphilum by Akberdin et al(Akberdin et al. 2018). All of the three modes of electron transfer during Methane oxidation (redox arm, direct coupling and uphill electron transfer) have been included in the models, since they are all possible and there is no definitive conclusion of which organism prefers what mode of methane oxidation.

\section{Metabolic Model Curation}

Draft models for both Methylobacter and Methylomonas underwent an extensive manual curation process, including chemical and charge-balancing, elimination of thermodynamically infeasible cycles, and ensuring network connectivity. Reactions in the draft model reaction set imbalanced in protons were checked with their appropriate protonation states and corrected by adding and deleting proton(s) on either side of the reaction equation. The remaining imbalanced reactions were stoichiometrically inaccurate and required the atoms on both sides of the reaction equation to be balanced. The metabolites consumed and produced by models were established by referring to literature and known transporter information (Boden et al. 2011; Caspi et al. 2016; Elbourne et al. 2017; Henry et al. 2010; Kanehisa 2008; Nguyen et al. 2018; Orata et al. 2018; Svenning et al. 2011; Szklarczyk et al. 2017; UniProt Consortium 2018; Wartiainen et al. 2006).

Genome annotations of methanotrophs indicate that formaldehyde assimilation can happen through the oxidative Pentose Phosphate Pathway as well as the formaldehyde/formate dehydrogenases and the tetrahydrofolate-associated pathways (Cai et al. 2016; Khmelenina et al. 2018). However, there have been extensive evidences that the Ribulose Monophosphate (RuMP) cycle is the major route for formaldehyde assimilation (Fu et al. 2017; He et al. 2020; Peyraud et

Peer) reviewing PDF | (2020:02:45973:2:0:NEW 28 May 2020) 
al. 2011). Also, multiple studies indicate that formaldehyde assimilation through the Serine cycle is insignificant (de la Torre et al. 2015; Fu et al. 2017; He et al. 2020). Based on transcriptomic data and metabolic flux measurements(de la Torre et al. 2015; Fu et al. 2017), we restricted the distribution of formaldehyde assimilation between the RuMP cycle and the tetrahydrofolateassociated pathways. In addition, the oxygen stoichiometry in pathways related to direct coupling methane oxidation and the coupling with cytochrome c oxidase have been set ( $1 \mathrm{~mol}$ Oxygen/mol pyrroloquinoline quinone and $0.5 \mathrm{~mol}$ Oxygen $/ \mathrm{mol}$ Cytochrome c) according to literature (de la Torre et al. 2015; Fu et al. 2017; Lieven et al. 2018; Sugioka et al. 1988).

While applying mass balance constraints to genome-scale metabolic models can display the net accumulation and consumption of metabolites within each microbial model, it fails to account for the regulation of reaction fluxes. The limitation of this constraint is better elucidated when focusing on reaction cycles that do not consume and produce metabolites. Because of the absence of metabolite consumption and production, the overall thermodynamic driving force of the cycles become zero and the cycle is incapable of supporting any net flux, and thus deemed thermodynamically and biologically infeasible (Schellenberger, Lewis \& Palsson, 2011). These thermodynamically infeasible cycles in our models were identified by inhibiting all nutrient uptakes to the cell and utilizing the optimization framework, Flux Variability Analysis (FVA), which maximizes and minimizes each reaction flux within the model based on the mass balance constraints (Mahadevan \& Schilling, 2003). Reactions whose fluxes reached the defined lower and upper bounds were determined to be unbounded reactions, group together based on stoichiometry, and systematically corrected. These cycles were eliminated by removing duplicate reactions, turning off lumped reactions, fixing reaction directionality, or selectively turning reactions on or off based on cofactor specificities found from literature (Ishida et al., 1969; Dekker, Lane \& Shapley, 1971; Chen, Lee \& Chang, 1991; Schomburg \& Stephan, 1995; Achterholt, Priefert \& Steinbuchel, 1998; Hadfield et al., 1999; Hutter \& Singh, 1999; Kai, Matsumura \& Izui, 2003; Feist et al., 2007; Dean, 2012; Flamholz et al., 2012; Lin et al., 2014; Christensen et al., 2017). The FVA optimization algorithm is shown below.

$$
\text { Maximize }_{\text {minimize }}\left(v_{j}\right) \quad v_{j}
$$
subject to

$\sum_{j \in J} S_{i j} \cdot v_{j}=0 \quad \forall i \in I$
$L B_{j} \leq v_{j} \leq U B_{j} \quad \forall j \in I$
$v_{\text {biomass }}=v_{\text {app }- \text { obj, threshold }}$

Both metabolic models were checked for the ability to produce biomass and metabolites they were known to produce (Svenning et al., 2011; Kalyuzhnaya et al., 2015). The metabolic functionalities of the models were ensured by identifying and manually adding reactions from biochemical databases, such as KEGG (Kanehisa \& Goto, 2000) and Uniprot (Consortium, 2018), to each model. Fully developed models of related organisms such as Methylococcus capsulatus and Methanomonas methanica for both Methylobacter and Methylomonas were utilized in help 
272

273

274

275

276

277

278

279

280

281

282

283

284

285

286

287

288

289

290

291

292

293

294

295

296

297

298

299

300

301

302

303

304

pinpoint absent enzymatic activity within our models. The addition of these reactions was confirmed using the bioinformatic algorithm, BLAST, which compares the genes of our organisms and related organisms and determines whether they are found to be orthologous. It was then tested whether these reactions would increase the number of thermodynamically infeasible cycles and promote for further curation of the models.

\section{Community model formation}

Following the curation of each individual microbial model, both were implemented to form a community model using the bi-level multi-objective optimization framework OptCom (Zomorrodi \& Maranas, 2012). OptCom simultaneously optimizes each individual community member's flux balance analysis problem as an inner-level optimization problem and the community model objective as an outer-level optimization problem. The maximization of the community biomass was set as the community objective function. The mathematical framework of the OptCom procedure is the following.

$$
\text { Maximize (or minimize) } \quad z=\text { Community level objective }
$$
subject to,

$$
\begin{aligned}
& \begin{array}{cc}
\multicolumn{2}{c}{\text { subject to, }} \\
v_{j}^{m a x i m i z e} & v_{\text {biomass }} \\
\text { subject to, } &
\end{array} \\
& \sum_{j \in J^{k}} S_{i j}^{k} \cdot v_{j}^{k}=0 \quad \forall i \in I^{k} \\
& L B_{j}^{k} \leq v_{j}^{k} \leq U B_{j}^{k} \quad \forall j \in J^{k} \\
& \underset{k}{v_{\text {uptake, } i}}=r_{\text {uptake, } i}^{k} \quad \forall i \in I_{\text {uptake }}^{k} \\
& \begin{array}{c}
k \\
v_{\text {export }, i}=r_{\text {export }, i}^{k} \quad \forall i \in I_{\text {export }}^{k}
\end{array} \\
& \sum_{k} r_{\text {uptake }, i}^{k}+e_{i}^{c}=\sum_{k} r_{\text {export }, i}^{k}+u_{i}^{c} \quad \forall i \in I_{\text {shared }} \\
& r_{\text {uptake, }, i}^{k}, \underset{\text { export, }, i}{k}, e_{i}^{c}, u_{i}^{c} \geq 0 \\
& \forall i \in I_{\text {shared }}
\end{aligned}
$$

The inner-level optimization problem(s) represents the steady-state FBA problem for each community member $k$ and limits the exchange (uptake and export) fluxes of shared metabolites between the individual species using the outer-level optimization problem parameters $r^{k}{ }_{u p t a k e, i}$ and $r_{\text {export, } i}^{k}$, respectively. The outer-level optimization problem constraint characterizes the mass balance for every shared metabolite in the extracellular environment within the shared metabolite pool. Metabolic interactions, with constraints, were confirmed with prior experimental research involving both community members or one member with a related organism found in the Lake Washington community (Yu et al., 2016).

\section{Formaldehyde Inhibition Constraint}

Formaldehyde production can be inhibitory towards the growth of Methylomonas and Methylobacter (Bussmann, Rahalkar \& Schink, 2006). Methylobacter is able to uptake formaldehyde excreted from Methylomonas to alleviate the inhibitory effects on Methylomonas growth. A constraint based upon the minimum and maximum inhibitory concentrations of formaldehyde was implemented to simulate formaldehyde's inhibitory effects on Methylobacter 
305 (Hou, Laskin \& Patel, 1979; Costa et al., 2001). While formaldehyde production is not forced in 306 the model simulations, this constraint accounts for the resulting growth inhibition if there is any 307 amount of formaldehyde excreted. The minimum formaldehyde concentration required for growth 308 inhibition was found to be $1 \mathrm{mM}$ (Hou, Laskin \& Patel, 1979) and the formaldehyde concentration 309 required for total growth inhibition (maximum formaldehyde concentration) was found to be 7 mM (Costa et al., 2001).

$$
v_{\text {biomass }}^{\text {Methylobacter }} \leq v_{\text {max biomass }}^{\text {Methylobacter }} \times\left(1-R \times v_{\text {formaldehyde uptake }}\right)
$$

The parameter $R$ was derived by finding the rate of change of biomass growth by the change in formaldehyde concentration.

\section{Bridging metabolic network gaps}

Automated draft reconstructions are limited as many reaction networks possess gaps due to missing reactions and blocked reactions. These are defined as reactions that lack production/consumption of its reactants/products. Major metabolic pathways were added based upon the literature of each organism (Kalyuzhnaya et al., 2015). Gaps were filled by referencing genetically related organisms to find missing metabolic capabilities. The presence of these possible reactions in the models were validated by cross referencing the relevant amino acid sequences between the reference organism and our models via pBlast. The reactions were then checked for the formation of thermodynamically infeasible cycles before being accepted.

\section{Computational resources}

The General Algebraic Modeling System (GAMS) version 24.8.5 with IBM CPLEX solver was utilized to run the optimization algorithms. The optimization frameworks were scripted in GAMS and then run on a Linux-based high-performance cluster computing system at the University of Nebraska-Lincoln. The downloaded models from ModelSEED with curations were parsed from System-Biology Markup Language (SBML) level 2 documentation using general-purpose programming language Python to generate the input files necessary for GAMS and subsequent manual curations.

\section{Results}

\section{Genome-scale metabolic models of Methylobacter and Methylomonas}

The draft genome-scale models of Methylobacter and Methylomonas are reconstructed using the ModelSEED database (Henry et al., 2016). The manual curation process ensures that there is no chemical and charge imbalance present in either of the models and there is no reaction with unrealistically high fluxes (infeasible reactions) without any nutrient uptake. The manual curation also reconnects a significant number of blocked metabolites to the network in both models (i.e., 107 metabolites for Methylobacter and 109 metabolites for Methylomonas). This enhancement of network connectivity is performed using available literature pertaining to major metabolic pathways that are known to be present in both the microbes (Kanehisa \& Goto, 2000; Kalyuzhnaya et al., 2015). The draft models were lacking some reactions in the important metabolic pathways i.e., the methane oxidation, pentose phosphate pathway, nitrogen fixation, cofactor, and amino acid production, which are curated at this stage. The model statistics are shown in Table 1. 
350

351

352

353

354

355

356

357

358

359

360

361

362

363

364

365

366

367

368

369

370

371

372

373

374

375

376

377

378

379

380

381

382

383

384

385

386

387

388

389

390

391

392

393

394

Supplementary Data S2 and S3 contain the model files for Methylobacter and Methylomonas, respectively.

Table 1: Model statistics for Methylobacter and Methylomonas

\section{Community dynamics under variable environmental conditions}

The individual models are integrated into a community model using available multi-objective computational optimization framework (Zomorrodi \& Maranas, 2012). The metabolic interactions between Methylobacter and Methylomonas are described using inter-organisms flow constraints. The community, as a whole, consumes methane, oxygen, and nitrogen, which is then shared between Methylobacter and Methylomonas. In addition, Methylobacter consumes formaldehyde produced by Methylomonas under certain conditions, which alleviates the formaldehyde toxicity on Methylomonas growth (Bussmann, Rahalkar \& Schink, 2006). At the same time, both Methylobacter and Methylomonas export carbon di-oxide to the environment. The community is simulated under four conditions in which methane, oxygen, and nitrogen are supplied to the community at various levels. These conditions are denoted as A, B, C, and D in Table 2 and correspond to the panels in Figure 1. It should be noted that the amount of each nutrient consumed by the members are not necessarily equal to the amount of nutrient supplied as only one of the nutrients acts as a limiting reagent in each limiting condition. The community model is illustrated in Figure 1.

Table 2: High and limiting nutrient conditions for community simulation.

Figure 1. Community dynamics showing the fluxes of key shared metabolites and biomass in the community model. A) High nutrient condition; B) Oxygen limited condition; C) Nitrogen limited condition; and D) Carbon limited condition

The community biomass flux is the highest when all the nutrients are highly abundant (Figure 1A). Methylomonas dominates the community in nutrient-rich condition. It is observed that a limited uptake of oxygen also imposes some restriction on the carbon and nitrogen utilization by the community, which results in reduced biomass fluxes for both Methylomonas and Methylobacter (Figure 1B). When oxygen uptake is limited, methane utilization by Methylomonas is still higher compared to Methylobacter despite an overall decrease in methane consumption by the community. An overall conservative nature of metabolism is observed in the community, as manifested by the least amount of carbon-di-oxide production and no formaldehyde production. In nitrogen-limited growth (Figure 1C), Methane uptake by Methylomonas decreases further and a high rate of respiration is observed, with the highest production of carbon-di-oxide. In Methanelimited condition (Figure 1D), while the metabolism in Methylobacter remains mostly unaffected, Methylomonas growth is severely hindered by the scarcity of Methane. In all the nutrient-limited conditions, Methylobacter is observed to dominate the community. The observations from Optcom simulations are consistent with the shifts in possible flux ranges under different conditions, as estimated by Flux Variability analysis. These observations indicate that the inherent degeneracy of metabolic fluxes in Flux Balance Analysis or Optcom does not affect the conclusions obtained in this work. The detailed results are presented in Supplementary Data S4. 
395

The total community biomass and community composition under methane and oxygen gradients are simulated to model the methane-oxygen counter gradient that exists in Lake Washington (Yu et al., 2016). In general, the total community biomass is observed to increase with both oxygen and methane uptake, as is expected from the increased abundance of nutrients. The community is completely dominated by Methylobacter under low methane/low oxygen conditions. An increase of methane at low oxygen conditions does not change the dominance of Methylobacter. Furthermore, the increase of oxygen under low methane conditions has minimal impact in changing the community composition, as can be seen from very slowly increasing ratio of Methylomonas to Methylobacter across the entire range of oxygen uptake at low methane uptake condition (Figure 2). The biggest charge in community composition is observed under high carbon and high oxygen conditions in which the community biomass is composed of $32 \%$ Methylobacter and 68\% Methylomonas, as compared to 99\% Methylobacter and 1\% Methylomonas in the low methane/low oxygen condition (Figure 2). The switch in community composition is consistent with the observations in nutrient rich condition. The complete numerical results are included in Supplementary Data S4.

Figure 2: The community composition and total biomass under varying Methane and Oxygen conditions. The size of each pie chart represents the total community biomass.

In nutrient-rich condition (shown in Figure 3), Methylobacter is the dominant community member and consumes the major portion of all of the shared the metabolites $i$. e., methane, oxygen, and nitrogen. Methylomonas consumes the major share of methane (80\%) and most of it is accumulated in biomass with a small amount of formaldehyde $(0.1 \mathrm{mmol} / \mathrm{gDC}$.hr $)$ being produced. Methylomonas also has a more active serine cycle converting formaldehyde into the metabolites in the central carbon metabolism. While the TCA cycle in both Methylobacter and Methylomonas is active in nutrient-rich condition, the activity of alpha-ketoglutarate dehydrogenase was very low.

Under the oxygen limiting condition (Figure 4), the flux through the methane oxidation pathway decreases by $20 \%$ in Methylobacter. Methylomonas consumes the majority (60\%) of the total methane uptaken by the community under the oxygen limiting condition (Figure 1B). Methylomonas diverts central carbon compounds to produce pyruvate via the assimilation of carbon di-oxide and acetaldehyde under the oxygen limited condition (Figure 4B). A small fraction of carbon di-oxide downstream of this reaction is secreted into the environment, which is the lowest among all the conditions. In this condition, Methylomonas has a complete TCA cycle activity.

Figure 3: Central carbon metabolism fluxes in Methylobacter (A) and Methylomonas (B) under high nutrient condition.

Figure 4: Central carbon metabolism fluxes in Methylobacter (A) and Methylomonas (B) under Oxygen limited condition.

The methane oxidizing pathway and TCA cycle reactions in Methylobacter do not show any significant change in nitrogen limited condition (Figure 5A). Methylobacter excretes more carbon di-oxide than nutrient-rich condition while consuming more oxygen than Methylomonas. On the other hand, the activity of the methane oxidation pathway of Methylomonas decreases (the lowest 
442 in any non-carbon limiting conditions). Furthermore, succinyl-CoA in Methylomonas is produced 443 from alpha-ketoglutarate in the TCA cycle, similar to oxygen limited conditions (Figure 5B). 444 Methylomonas, similar to Methylobacter, also excretes significantly high amount of carbon-di445 oxide in nitrogen-limited condition.

446

447

In the carbon limited growth condition, Methylomonas takes up very small amount of methane, while Methylobacter takes up most of the methane supplied to the community (Figure 6). The central metabolism of Methylobacter is not altered under this carbon limited condition (Figure 6A). However, in Methylomonas, carbon di-oxide is scavenged by assimilating succinyldihydrolipoamide and carbon di-oxide to succinyl-CoA (Figure 6B). This reaction is inactive in the oxygen limiting condition. Methylomonas also displays minimal activity in its serine cycle under carbon limiting condition.

Figure 5: Central carbon metabolism fluxes in Methylobacter (A) and Methylomonas (B) under Nitrogen limited condition.

Figure 6: Central carbon metabolism fluxes in Methylobacter (A) and Methylomonas (B) under Carbon limited condition.

\section{Dynamic shifts in metabolism under sediment incubated microcosm and synthetic co- culture composition}

Previous studies have shown inconsistencies between the microcosm incubated from Lake Washington sediments and synthetic community cultured in the lab. In the natural community (microcosm incubated from the lake sediments), Methylobacter has been shown to be dominant under both low methane/high oxygen and high methane/low oxygen conditions (Beck et al., 2013). However, Methylomonas outcompeted Methylobacter in both low methane/high oxygen and high methane/low oxygen conditions in synthetic co-cultures ( $\mathrm{Yu}$ et al., 2016). To elucidate the metabolic flux distributions and the extent of inter-species interactions that gives rise to the observed community composition in the two conditions, the experimentally observed species abundance ratio was imposed on the growth rates of Methylobacter (MB) and Methylomonas (ML) as a constraint in the community optimization framework. For the sediment-incubated community, a MB:ML ratio of 9.3:1.0 and for the synthetic community, a MB:ML ratio of 0.05:1.0 were used.

Figure 7: Flux distribution for select metabolites in Methylobacter and Methylomonas under A) Lake Washington sediment-incubated microcosm conditions and B) synthetic co-culture conditions.

The vast majority of reactions in Methylomonas has lower flux values in the sediment-incubated community compared to the synthetic community (Figure 7). This occurs because Methylomonas constitutes a smaller portion of the total community biomass in the sediment-incubated community. There are alternate pathways to produce pyruvate which has increased flux (Figure 7A). These pathways produce pyruvate by assimilating carbon di-oxide and acetaldehyde and by the assimilation of cysteine and mercaptopyruvate. However, Methylomonas produces less pyruvate overall even with the increased flux in these pathways, since other pyruvate producing pathways decrease in flux. Primarily, the flux through L-serine and ammonia assimilation to produce pyruvate is high in the synthetic condition but decreases in sediment-incubated condition. Under sediment-incubated conditions, Methylobacter uses methane as its primary carbon source and does not consume any formaldehyde produced by Methylomonas. On the other hand, 
489

490

491

492

493

494

495

496

497

498

499

500

501

502

503

504

505

506

507

508

509

510

511

512

513

514

515

516

517

518

519

520

521

522

523

524

525

526

527

528

529

530

531

532

533

Methylobacter takes up some of the secreted formaldehyde from Methylomonas as a carbon source in addition to Methane under the synthetic condition (Figure 7B).

\section{Discussion}

Aerobic methane oxidation in freshwater lakes around the world is a key metabolic process that significantly affects the carbon cycle by acting as a major sink of methane. Lake Washington provides an wonderful opportunity to study the methane cycling, with up to $20 \%$ of the organic carbon being released as methane through decomposition and consuming up to $10 \%$ of the dissolved oxygen in the lake water (Kuivila et al., 1988). With the goal to understand the dynamics of the methane cycling Lake Washington community, we integrated high-quality manually curated and refined genome-scale metabolic models of highly abundant, functionally important, and representative community members using multi-level optimization-based frameworks. While this community have been studied by many researchers in the previous years with in vivo tools like synthetic co-cultures, metagenomics, and metatranscriptomics (Hernandez et al., 2015; Oshkin et al., 2015; Yu et al., 2016; Krause et al., 2017), an in silico approach like the one used in this work is needed to have a deeper understanding of the underlying mechanisms that govern the interspecies interactions and in turn, the community structure, function and dynamics in Lake Washington. As the global carbon transactions are changing and the release of greenhouse gases in the atmosphere is consistently getting worse every year, it is imperative for us to put our best efforts in mitigating the harmful effects. To do that, the use of genome-scale metabolic modeling tools to understand how the microbial communities are involved in these metabolic processes function in natural environments is essential.

To make the community model a good representation of the naturally occurring methane cycling community, we selected two highly abundant and functionally important microbial species. Following the manual curation process of both metabolic models, it was found that Methylobacter was more efficient than Methylomonas at producing biomass when simulated under the same standard growth environment and biological constraints in the Lake Washington, except at highly nutrient-rich conditions. Since Methylobacter and Methylomonas are competitors for the sole carbon source, methane, the overall metabolic efficiency is an important factor in the methane utilization ratio of the two species. While there is no direct literature evidence that suggests that one of them is more efficient in utilizing methane for growth compared to the other, it is highly possible that they might be limited by other small molecules that inhibit high methane consumption. For example, the community was tested under carbon, oxygen, and nitrogen limited conditions to observe how its central metabolic pathway and the community composition varied, and we observe the dominance of Methylobacter in the community in all of the limited growth conditions (Figures 4 through 6) but the dominance of Methylomonas in the nutrient-rich condition. This is observed even during the oxygen limited growth condition, where Methylobacter is unable to consume as much methane as Methylomonas. In this condition, Methylomonas takes up more methane than Methylobacter but Methylobacter can still maintain its dominance.

Each of the nutrient limited conditions shows variable differences within the methane oxidation pathway, the serine cycle, and the TCA cycle. While, based on the simulation, we did not observe a noticeable flux through the serine cycle in either Methylomonas or Methylobacter in all nutrientlimited conditions, it was significantly active in Methylomonas in nutrient rich condition. In

Peer] reviewing PDF | (2020:02:45973:2:0:NEW 28 May 2020) 
534

535

536

537

538

539

540

541

542

543

544

545

546

547

548

549

550

551

552

553

554

555

556

557

558

559

560

561

562

563

564

565

566

567

568

569

570

571

572

573

574

575

576

577

578

579

oxygen limiting condition, the community was also observed to conserve as much resource as possible. For example, while Methylomonas excretes some amount of carbon dioxide in all conditions, it routes most of it back to central carbon metabolism through the direction reversal of pyruvate decarboxylase. Although there is currently no experimental studies pointing to this phenomenon, studies in other organisms suggest a high oxygen sensitivity of this enzyme (Eram \& Ma, 2013). Therefore, a possible explanation of the shifts in pyruvate metabolism is the oxygen sensitivity of this enzyme, which needs to be further studied.

In most of the simulation conditions, Methylobacter and Methylomonas were observed to assimilate most of the consumed carbon though the Entner-Doudoroff (ED) pathway. This was evident from the high flux of fructose-6-phospahte to 6-phophogluconate and thereafter pyruvate. In contrast, the Embden-Meyerhof-Parnas (EMP) variant of the glycolytic pathway was predicted to be the dominant pathway in some other methanotrophs like M. buryatense through model simulations (de la Torre et al. 2015; He et al. 2019; Kalyuzhnaya et al. 2013). It should be noted that studies measuring glycolytic fluxes experimentally in Methylobacter and Methylomonas species are sparse in literature. A recent ${ }^{13} \mathrm{C}$ tracer analysis by $\mathrm{Fu}$ et al suggested that the flux partition between EMP and ED variants is unresolved since they both manifest fully labeled downstream molecules (Fu et al. 2017). Another interesting observation from this study is the activity levels of alpha-ketoglutarate dehydrogenase enzyme in all conditions. The presence and expression of alpha-ketoglutarate dehydrogenase in methanotrophs has been a matter of debate for quite some time (Zhao \& Hanson, 1984; Theisen \& Murrell, 2005), which is manifested as the inability of methanotrophs to grow on multi-carbon substrates (Smith, Trotsenko \& Murrell, 2010). In this study we observe very negligible $(<2 \%$ of the TCA cycle flux) alpha-ketoglutarate dehydrogenase activity in all conditions. At this point it is not straightforward to decipher what exactly might be the regulating factor to this enzyme and warrants further experimentation.

Finally, the community model was simulated with fixed abundance ratios of the two members to reflect the composition in synthetic co-culture and sediment-incubated community. The changes in community composition as oxygen and carbon levels change are more consistent with behaviors in sediment samples than synthetic co-cultures (Hernandez et al., 2015; Yu et al., 2016). Methylobacter is able to utilize methane to produce biomass at a more efficient level than Methylomonas. However, when the synthetic co-culture condition is imposed on the species abundance ratio, i.e., favoring Methylomonas biomass, Methylobacter takes up formaldehyde produced by Methylomonas in addition to methane consumption, which allows it to bypass the oxygen-intensive reaction of oxidizing methane to methanol to some degree. This commensal relationship helps Methylobacter to enhance its biomass even when the uptake of the original carbon source (methane) is low while protecting Methylomonas from the toxicity and growth inhibitory effects of formaldehyde (Hou, Laskin \& Patel, 1979; Costa et al., 2001). In our optimization formulation, the inhibitory formaldehyde constraint placed on Methylobacter makes the consumption of formaldehyde detrimental towards biomass production, but it can simultaneously act as a carbon source and compensates for its inhibitory effects. This is contradictory to what was observed in the co-culture experiments ( $\mathrm{Yu}$ et al., 2016). Similar to formaldehyde, methanol export was also not observed in the simulations although some previous studies have reported ethanol production(Krause et al. 2017; Zheng et al. 2018). This lack of excretion of intermediate carbon molecules is often observed in steady-state Flux Balance Analysis models without any enzyme kinetic information, where pathways upstream of biomass consumes

Peer] reviewing PDF | (2020:02:45973:2:0:NEW 28 May 2020) 
580

581

582

583

584

585

586

587

588

589

590

591

592

593

594

595

596

597

598

599

600

601

602

603

604

605

606

607

608

609

610

611

612

613

614

615

616

617

618

619

620

621

622

623

624

625

most of the intermediates. However, it should be noted that, the synthetic co-culture experiments reported in literature involved other community members, whose metabolic interactions with Methylobacter and Methylomonas are not well characterized to date. Some studies have indicated co-operative relationships between Methylobacter and other microbial species from Methylophilaceae family (Beck et al., 2013), which might potentially impact that community dynamics in synthetic co-cultures. Moreover, despite the dominance of Methylomonas in the synthetic community at a species level (Soni et al., 1998; Hoefman et al., 2012), further assessment of community composition at a higher taxonomic level indicated a consistency with naturally observed composition ( $\mathrm{Yu}$ et al., 2016). We hypothesize that this discrepancy is possible, given that there is high functional redundancy present in Lake Washington community, similar to any naturally occurring microbial ecosystem (Galand et al., 2018; Louca et al., 2018; Islam et al., 2019; Jia \& Whalen, 2020).

\section{Conclusion}

In this work, we attempted to enhance our mechanistic understanding of the dynamics in the methane cycling Lake Washington community through genome-scale metabolic modeling of the representative and functionally important community members, Methylobacter tundripaludum 21/22 and Methylomonas sp LW13. The understanding of this community behavior will be a foundation for future studies that aim at the long-term goal of creating a complex synthetic community capable of carrying out certain desired functions through the consumption of methane, thus mitigating the harmful effects of methane release in the atmosphere. One should be aware of the fact that the in silico results need to be further tested and confirmed through relevant experiments before any engineering strategies can be successfully employed. The community metabolic model, in that regard, should be expanded to include other major players of the Lake Washington community, i.e., members of Bacteroidetes and Proteobacteria phyla. Including these organisms in our community metabolic model will enable us to explain currently unidentified inter-species metabolite exchanges/interactions that play important role in the cycling of methane as well as other nutrients.

\section{References}

Achterholt S, Priefert H, Steinbuchel A. 1998. Purification and Characterization of the Coniferyl Aldehyde Dehydrogenase from Pseudomonas sp. Strain HR199 and Molecular Characterization of the Gene. J Bacteriol 180:4387-4391.

Akberdin IR, Thompson M, Hamilton R, Desai N, Alexander D, Henard CA, Guarnieri MT, and Kalyuzhnaya MG. 2018. Methane utilization in Methylomicrobium alcaliphilum 20Z(R): a systems approach. Sci Rep 8:2512. 10.1038/s41598-018-20574-z

Alsiyabi A, Immethun CM, and Saha R. 2019. Modeling the Interplay between Photosynthesis, CO2 Fixation, and the Quinone Pool in a Purple Non-Sulfur Bacterium. Scientific Reports 9:12638. 10.1038/s41598-019-49079-z

Auman AJ, Stolyar S, Costello AM, Lidstrom ME. 2000. Molecular characterization of methanotrophic isolates from freshwater lake sediment. Applied and Environmental Microbiology. DOI: 10.1128/AEM.66.12.5259-5266.2000.

Beck DAC, Kalyuzhnaya MG, Malfatti S, Tringe SG, Glavina del Rio T, Ivanova N, Lidstrom ME, Chistoserdova L. 2013. A metagenomic insight into freshwater methane-utilizing communities and evidence for cooperation between the Methylococcaceae and the Methylophilaceae. PeerJ 1. DOI: 10.7717/peerj.23. 
626

627

628

629

630

631

632

633

634

635

636

637

638

639

640

641

642

643

644

645

646

647

648

649

650

651

652

653

654

655

656

657

658

659

660

661

662

663

664

665

666

667

668

669

670
Bizukojc M, Dietz D, Sun J, Zeng A. 2010. Metabolic modelling of syntrophic-like growth of a 1,3-propanediol producer, Clostridium butyricum, and a methanogenic archeon, Methanosarcina mazei, under anaerobic conditions. Bioprocess Biosyst Eng 33:507-523. DOI: 10.1007/s00449-009-0359-0.

Boden R, Cunliffe M, Scanlan J, Moussard H, Kits KD, Klotz MG, Jetten MS, Vuilleumier S, Han J, Peters L, Mikhailova N, Teshima H, Tapia R, Kyrpides N, Ivanova N, Pagani I, Cheng JF, Goodwin L, Han C, Hauser L, Land ML, Lapidus A, Lucas S, Pitluck S, Woyke T, Stein L, and Murrell JC. 2011. Complete genome sequence of the aerobic marine methanotroph Methylomonas methanica MC09. J Bacteriol 193:7001-7002. 10.1128/JB.06267-11

Bordel S, Rodriguez Y, Hakobyan A, Rodriguez E, Lebrero R, and Munoz R. 2019a. Genome scale metabolic modeling reveals the metabolic potential of three Type II methanotrophs of the genus Methylocystis. Metab Eng 54:191-199. 10.1016/j.ymben.2019.04.001

Bordel S, Rojas A, and Munoz R. 2019b. Reconstruction of a Genome Scale Metabolic Model of the polyhydroxybutyrate producing methanotroph Methylocystis parvus OBBP. Microb Cell Fact 18:104. 10.1186/s12934-019-1154-5

Borenstein E, Feldman MW. 2009. Topological Signatures of Species Interactions in Metabolic Networks. Journal of Computational Biology 16. DOI: 10.1089/cmb.2008.06TT.

Bussmann I, Rahalkar M, Schink B. 2006. Cultivation of methanotrophic bacteria in opposing gradients of methane and oxygen. FEMS Microbiology Ecology 56:331-344. DOI: 10.1111/j.1574-6941.2006.00076.x.

Cai Y, Zheng Y, Bodelier PL, Conrad R, and Jia Z. 2016. Conventional methanotrophs are responsible for atmospheric methane oxidation in paddy soils. Nat Commun 7:11728. 10.1038/ncomms 11728

Carere CR, McDonald B, Peach HA, Greening C, Gapes DJ, Collet C, and Stott MB. 2019. Hydrogen Oxidation Influences Glycogen Accumulation in a Verrucomicrobial Methanotroph. Front Microbiol 10. 10.3389/fmicb.2019.01873

Caspi R, Billington R, Ferrer L, Foerster H, Fulcher CA, Keseler IM, Kothari A, Krummenacker M, Latendresse M, Mueller LA, Ong Q, Paley S, Subhraveti P, Weaver DS, and Karp PD. 2016. The MetaCyc database of metabolic pathways and enzymes and the BioCyc collection of pathway/genome databases. Nucleic Acids Res 44:D471-480. 10.1093/nar/gkv1164

Chan SHJ, Simons MN, Maranas CD. 2017. SteadyCom: Predicting microbial abundances while ensuring community stability. PLoS Computational Biology 13. DOI: 10.1371/journal.pcbi.1005539.

Chen Z, Lee WR, Chang SH. 1991. https://febs.onlinelibrary.wiley.com/doi/full/10.1111/j.14321033.1991.tb16371.x. European Journal of Biochemistry 202:263-267. DOI:

10.1111/j.1432-1033.1991.tb16371.x.

Christensen EM, Patel SM, Korasick DA, Campbell AC, Krause KL, Becker DF, Tanner JJ. 2017. Resolving the Cofactor Binding Site in the Proline Biosynthetic Enzyme Human Pyrroline-5-Carboxylate Reductase 1. Journal of Biological Chemistry. DOI: 10.1074/jbc.M117.780288.

Consortium TU. 2018. UniProt: a worldwide hub of protein knowledge. Nucleic Acids Research 47:D506-D515. DOI: 10.1093/nar/gky1049.

Costa C, M V, Dijkema C, Stams A. 2001. The effect of oxygen on methanol oxidation by an obligate methanotrophic bacterium studied by in vivo (13)C nuclear magnetic resonance spectroscopy. J Ind Microbiol Bictechnol 26:9-14. DOI: 10.1038/sj/jim/7000075.

Peer] reviewing PDF | (2020:02:45973:2:0:NEW 28 May 2020) 
671

672

673

674

675

676

677

678

679

680

681

682

683

684

685

686

687

688

689

690

691

692

693

694

695

696

697

698

699

700

701

702

703

704

705

706

707

708

709

710

711

712

713

714

715

716

717

Cui M, Ma A, Qi H, Zhuang X, and Zhuang G. 2015. Anaerobic oxidation of methane: an "active" microbial process. Microbiologyopen 4:1-11. 10.1002/mbo3.232

de la Torre A, Metivier A, Chu F, Laurens LM, Beck DA, Pienkos PT, Lidstrom ME, and Kalyuzhnaya MG. 2015. Genome-scale metabolic reconstructions and theoretical investigation of methane conversion in Methylomicrobium buryatense strain 5G(B1). Microb Cell Fact 14:188. 10.1186/s12934-015-0377-3

Dean L. 2012. Methylenetetrahydrofolate Reductase Deficiency. Medical Genetics Summaries.

Dekker EE, Lane RS, Shapley A. 1971. 2-Keto-4-hydroxybutyrate aldolase. Identification as 2keto-4-hydroxyglutarate aldolase, catalytic properties, and role in the mammalian metabolism of L-homoserine. Biochemistry 10:1353-1364. DOI: 10.1021/bi00784a013.

Elbourne LD, Tetu SG, Hassan KA, and Paulsen IT. 2017. TransportDB 2.0: a database for exploring membrane transporters in sequenced genomes from all domains of life. Nucleic Acids Res 45:D320D324. 10.1093/nar/gkw1068

Eram MS, Ma K. 2013. Decarboxylation of pyruvate to acetaldehyde for ethanol production by hyperthermophiles. Biomolecules 3:578-596. DOI: 10.3390/biom3030578.

Feist AM, Henry, Christopher S, Reed JL, Krummenacker M, Joyce AR, Karp PD, Broadbelt LJ, Hatzimanikatis V, Palsson BO. 2007. A genome-scale metabolic reconstruction for Escherichia coli K-12 MG1655 that accounts for 1260 ORFs and thermodynamic information. Mol Syst Biol 3:121. DOI: 10.1038/msb4100155.

Flamholz A, Noor E, Bar-Even A, Milo R. 2012. eQuilibrator - the biochemical thermodynamics calculator. Nucleic Acids Res 40:D770-D775. DOI: 10.1093/nar/gkr874.

Freilich S, Kreimer A, Borenstein, Elhanan, Yosef N, Sharan R, Gophna U, Ruppin E. 2009. Metabolic-network-driven analysis of bacterial ecological strategies. Genome Biol 10. DOI: 10.1186/gb-2009-10-6-r61.

Frey E. 2010. Evolutionary game theory: Theoretical concepts and applications to microbial communities. Physica A: Statistical Mechanics and its Applications 389:4265-4298. DOI: 10.1016/j.physa.2010.02.047.

$\mathrm{Fu} \mathrm{Y,} \mathrm{Li} \mathrm{Y,} \mathrm{and} \mathrm{Lidstrom} \mathrm{M.} \mathrm{2017.} \mathrm{The} \mathrm{oxidative} \mathrm{TCA} \mathrm{cycle} \mathrm{operates} \mathrm{during} \mathrm{methanotrophic}$ growth of the Type I methanotroph Methylomicrobium buryatense 5GB1. Metab Eng 42:4351. 10.1016/j.ymben.2017.05.003

Galand PE, Pereira O, Hochart C, Auguet JC, Debroas D. 2018. A strong link between marine microbial community composition and function challenges the idea of functional redundancy. The ISME Journal 12:2470-2478. DOI: 10.1038/s41396-018-0158-1.

Hadfield A, Kryger G, Ouyang J, Petsko GA, Ringe D, Viola R. 1999. Structure of Aspartate- $\beta$ semialdehyde Dehydrogenase from Escherichia coli, a Key Enzyme in the Aspartate Family of Amino Acid Biosynthesis. Journal of Molecular Biology 289:991-1002. DOI: 10.1006/jmbi.1999.2828.

Hanson RS, Hanson TE. 1996. Methanotrophic bacteria. Microbiological Reviews. DOI: 10.1002/0471263397.env316.

He H, Noor E, Ramos-Parra PA, Garcia-Valencia LE, Patterson JA, Diaz de la Garza RI, Hanson AD, and Bar-Even A. 2020. In Vivo Rate of Formaldehyde Condensation with Tetrahydrofolate. Metabolites 10. 10.3390/metabo10020065

$\mathrm{He}$ L, Fu Y, and Lidstrom ME. 2019. Quantifying Methane and Methanol Metabolism of "Methylotuvimicrobium buryatense" 5GB1C under Substrate Limitation. mSystems 4. 10.1128/mSystems.00748-19

Henry CS, Bernstein HC, Weisenhorn P, Taylor RC, Lee J-Y, Zucker J, Song H-S. 2016.

PeerJ reviewing PDF | (2020:02:45973:2:0:NEW 28 May 2020) 
718

Microbial Community Metabolic Modeling: A Community Data-Driven Network

Reconstruction. J Cell Physiol 231:2339-2345. DOI: 10.1002/jcp.25428.

Henry CS, DeJongh M, Best AA, Frybarger PM, Linsay B, Stevens RL. 2010. High-throughput generation, optimization and analysis of genome-scale metabolic models. Nat Biotechnol 28:977-982. DOI: 10.1038/nbt.1672.

Hernandez ME, Beck, David A.C., Lidstrom ME, Chistoserdova L. 2015. Oxygen availability is a major factor in determining the composition of microbial communities involved in methane oxidation. PeerJ 3. DOI: 10.7717/peerj.801.

Hoefman S, van der Ha D, De Vos P, Boon N, Heylen K. 2012. Miniaturized extinction culturing is the preferred strategy for rapid isolation of fast-growing methane-oxidizing bacteria. Microbial Biotechnology 5:368-378. DOI: 10.1111/j.1751-7915.2011.00314.x.

Hou CT, Laskin AI, Patel RN. 1979. Growth and Polysaccharide Production by Methylocystis parvus OBBP on Methanol. Appl Environ Microbiol 37:800-804.

Houghton JT, Jenkins GJ, Ephraums JJ. 1990. Climate change: the IPCC scientific assessment. Climate change: the IPCC scientific assessment. DOI: 10.2307/1971875.

Hutter B, Singh M. 1999. Properties of the $40 \mathrm{kDa}$ antigen of Mycobacterium tuberculosis, a functional L-alanine dehydrogenase. Biochem J 343:669-672.

IPCC. 2013. AR5 - Summary for Policymakers. DOI: 10.1017/CBO9781107415324.Summary.

Ishida Y, Shirafuji H, Kida M, Yoneda M. 1969. Studies on the Guanosine Degrading System in Bacterial Cell. Agricultural and Biological Chemistry 33:384-390. DOI: 10.1080/00021369.1969.10859324.

Islam MM, Fernando SC, Saha R, Saha R. 2019. Metabolic Modeling Elucidates the Transactions in the Rumen Microbiome and the Shifts upon Virome Interactions.

Islam MM, Saha R. 2018. Computational Approaches on Stoichiometric and Kinetic Modeling for Efficient Strain Design. New York, NY: Humana Press. DOI: 10.1007/978-1-49397295-1_5.

Islam MM, Thomas VC, Van Beek M, Ahn JS, Alqarzaee AA, Zhou C, Fey PD, Bayles KW, and Saha R. 2020. An integrated computational and experimental study to investigate Staphylococcus aureus metabolism. NPJ Syst Biol Appl 6:3. 10.1038/s41540-019-0122-3

Jia Y, Whalen JK. 2020. A new perspective on functional redundancy and phylogenetic niche conservatism in soil microbial communities. Pedosphere 30:18-24. DOI: 10.1016/S10020160(19)60826-X.

Kai Y, Matsumura H, Izui K. 2003. Phosphoenolpyruvate carboxylase: three-dimensional structure and molecular mechanisms. Arch Biochem Biophys 414:170-179. DOI: 10.1016/s0003-9861(03)00170-x.

Kalyuzhnaya MG, Lapidus A, Ivanova N, Copeland AC, McHardy AC, Szeto E, Salamov A, Grigoriev IV, Suciu D, Levine SR, Markowitz VM, Rigoutsos I, Tringe SG, Bruce DC, Richardson PM, Lidstrom ME, and Chistoserdova L. 2008. High-resolution metagenomics targets specific functional types in complex microbial communities. Nat Biotechnol 26:10291034. 10.1038/nbt.1488

Kalyuzhnaya MG, Yang S, Rozova ON, Smalley NE, Clubb J, Lamb A, Gowda GA, Raftery D, Fu Y, Bringel F, Vuilleumier S, Beck DA, Trotsenko YA, Khmelenina VN, and Lidstrom ME. 2013. Highly efficient methane biocatalysis revealed in a methanotrophic bacterium. Nat Commun 4:2785. 10.1038/ncomms3785

Kalyuzhnaya MG, Lamb AE, McTaggart TL, Oshkin IY, Shapiro N, Woyke T, Chistoserdova L. 2015. Draft Genome Sequences of Gammaproteobacterial Methanotrophs Isolated from 
Lake Washington Sediment. Genome Announcements 3:e00103-15. DOI: 10.1128/genomeA.00103-15.

Kanehisa M, Goto S. 2000. KEGG: Kyoto Encyclopedia of Genes and Genomes. Nucleic Acids Research 28:27-30. DOI: 10.1093/nar/28.1.27.

Khmelenina VN, Colin Murrell J, Smith TJ, and Trotsenko YA. 2018. Physiology and Biochemistry of the Aerobic Methanotrophs. In: Rojo F, ed. Aerobic Utilization of Hydrocarbons, Oils and Lipids. Cham: Springer International Publishing, 1-25.

Krause SMB, Johnson T, Karunaratne YS, Fu Y, Beck DAC, Chistoserdova L, Lidstrom ME. 2017. Lanthanide-dependent cross-feeding of methane-derived carbon is linked by microbial community interactions. Proceedings of the National Academy of Sciences of the United States of America. DOI: 10.1073/pnas.1619871114.

Kuivila KM, Murray JW, Devol AH, Lidstrom ME, Reimers CE. 1988. Methane cycling in the sediments of Lake Washington. Limnology and Oceanography 33:571-581. DOI: 10.4319/1o.1988.33.4.0571.

Lehmann L, Keller L. 2006. The evolution of cooperation and altruism - a general framework and a classification of models. Journal of Evolutionary Biology 19:1365-1376. DOI: 10.1111/j.1420-9101.2006.01119.x.

Lewis N, Schramm G, Bordbar A, Schellenberger J, Andersen M, Cheng J, Patel N, Yee A, Lewis R, Eils R, Konig R, Palsson BO. 2010. Large-scale in silico modeling of metabolic interactions between cell types in the human brain. Nature Biotechnology 28:1279-1285. DOI: $10.1038 /$ nbt.1711.

Lieven C, Petersen LAH, Jorgensen SB, Gernaey KV, Herrgard MJ, and Sonnenschein N. 2018. A Genome-Scale Metabolic Model for Methylococcus capsulatus (Bath) Suggests Reduced Efficiency Electron Transfer to the Particulate Methane Monooxygenase. Front Microbiol 9:2947. 10.3389/fmicb.2018.02947

Lin X, Tfaily MM, Green SJ, Steinweg JM, Chanton P, Imvittaya A, Chanton JP, Cooper W, Schadt C, Kostka JE. 2014. Microbial Metabolic Potential for Carbon Degradation and Nutrient (Nitrogen and Phosphorus) Acquisition in an Ombrotrophic Peatland. Applied and Environmental Microbiology 80:3531 LP - 3540. DOI: 10.1128/AEM.00206-14.

Louca S, Polz MF, Mazel F, Albright MBN, Huber JA, O’Connor MI, Ackermann M, Hahn AS, Srivastava DS, Crowe SA, Doebeli M, Parfrey LW. 2018. Function and functional redundancy in microbial systems. Nature Ecology \& Evolution 2:936-943. DOI: 10.1038/s41559-018-0519-1.

Magnúsdóttir S, Heinken A, Kutt L. 2017. Generation of genome-scale metabolic reconstructions for 773 members of the human gut microbiota. Nature Biotechnology 35:81-89. DOI: 10.1038/nbt.3703.

Mahadevan R, Schilling C. 2003. Models., The effects of alternate optimal solutions in constraint-based genome-scale metabolic. Metab Eng 5:264-76. DOI:

10.1016/j.ymben.2003.09.002.

Maranas CD, Zomorrodi AR. 2016. Optimization Methods in Metabolic Networks. Wiley.

Mohammadi SS, Schmitz RA, Pol A, Berben T, Jetten MSM, and Op den Camp HJM. 2019. The Acidophilic Methanotroph Methylacidimicrobium tartarophylax 4AC Grows as Autotroph on H2 Under Microoxic Conditions. Front Microbiol 10. 10.3389/fmicb.2019.02352

Nadell CD, Foster KR, Xavier JB. 2010. Emergence of Spatial Structure in Cell Groups and the Evolution of Cooperation. PLoS Computational Biology 6:e1000716. DOI: 10.1371/journal.pcbi. 1000716 . 
810

811

812

813

814

815

816

817

818

819

820

821

822

823

824

825

826

827

828

829

830

831

832

833

834

835

836

837

838

839

840

841

842

843

844

845

846

847

848

849

850

851

852

853

854

855

Naizabekov S, and Lee YE. 2020. Genome-Scale Metabolic Model Reconstruction and in Silico Investigations of Methane Metabolism in Methylosinus trichosporium OB3b. Microorganisms 8. 10.3390/microorganisms 8030437

Nisbet EG, Manning MR, Dlugokencky EJ, Fisher RE, Lowry D, Michel SE, Myhre CL, Platt SM, Allen G, Bousquet P, Brownlow R, Cain M, France JL, Hermansen O, Hossaini R, Jones AE, Levin I, Manning AC, Myhre G, Pyle JA, Vaughn BH, Warwick NJ, White JWC. 2019. Very Strong Atmospheric Methane Growth in the 4 Years 2014-2017: Implications for the Paris Agreement. Global Biogeochemical Cycles. DOI: 10.1029/2018GB006009.

Nguyen NL, Yu WJ, Gwak JH, Kim SJ, Park SJ, Herbold CW, Kim JG, Jung MY, and Rhee SK. 2018. Genomic Insights Into the Acid Adaptation of Novel Methanotrophs Enriched From Acidic Forest Soils. Front Microbiol 9:1982. 10.3389/fmicb.2018.01982

Orata FD, Kits KD, and Stein LY. 2018. Complete Genome Sequence of Methylomonas denitrificans Strain FJG1, an Obligate Aerobic Methanotroph That Can Couple Methane Oxidation with Denitrification. Genome Announc 6. 10.1128/genomeA.00276-18

Orth JD, Thiele I, Palsson BO. 2010. What is flux balance analysis? Nature Biotechnology 28:245-248. DOI: 10.1038/nbt.1614.

Oshkin IY, Beck DA, Lamb AE, Tchesnokova V, Benuska G, McTaggart TL, Kalyuzhnaya MG, Dedysh SN, Lidstrom ME, Chistoserdova L. 2015. Methane-fed microbial microcosms show differential community dynamics and pinpoint taxa involved in communal response. ISME J 9:1119-1129. DOI: 10.1038/ismej.2014.203.

Peyraud R, Schneider K, Kiefer P, Massou S, Vorholt JA, and Portais JC. 2011. Genome-scale reconstruction and system level investigation of the metabolic network of Methylobacterium extorquens AM1. BMC Syst Biol 5:189. 10.1186/1752-0509-5-189

De Roy K, Marzorati M, Van den Abbeele P, Van de Wiele T, Boon N. 2014. Synthetic microbial ecosystems: an exciting tool to understand and apply microbial communities. Environmental Microbiology 16:1427-1481. DOI: 10.1111/1462-2920.12343.

Schellenberger J, Lewis N, Palsson BO. 2011. Elimination of thermodynamically infeasible loops in steady-state metabolic models. Biophys $J$ 100:544-553. DOI: 10.1016/j.bpj.2010.12.3707.

Schomburg D, Stephan D. 1995. 2-Hydroxy-3-oxopropionate reductase. Enzyme Handbook 9. DOI: $10.1007 / 978-3-642-85200-8 \_60$.

Schroeder WL, Harris SD, and Saha R. 2020. Computation-driven analysis of model polyextremotolerant fungus Exophiala dermatitidis: defensive pigment metabolic costs and human applications. iScience. https://doi.org/10.1016/j.isci.2020.100980

Schroeder WL, and Saha R. 2019. A Computational Framework to Study the Primary Lifecycle Metabolism of $<$ em $>$ Arabidopsis thaliana $</$ em $>$. bioRxiv:761189. 10.1101/761189

Shoaie S, Nielsen J. 2014. Elucidating the interactions between the human gut microbiota and its host through metabolic modeling. Front Genet 5:86. DOI: 10.3389/fgene.2014.00086.

Shou W, Ram S, Vilar JMG. 2007. Synthetic cooperation in engineered yeast populations. Proceedings of the National Academy of Sciences of the United States of America 104:1877-1882. DOI: 10.1073/pnas.0610575104.

Smith TJ, Trotsenko YA, Murrell JC. 2010. Physiology and Biochemistry of the Aerobic Methane Oxidizing Bacteria BT - Handbook of Hydrocarbon and Lipid Microbiology. In: Timmis KN ed. Berlin, Heidelberg: Springer Berlin Heidelberg, 765-779. DOI: 10.1007/978-3-540-77587-4_58. 
856

857

858

859

860

861

862

863

864

865

866

867

868

869

870

871

872

873

874

875

876

877

878

879

880

881

882

883

884

885

886

887

888

889

890

891

892

893

894

895

896

897

898

899

900

901

902

Soni BK, Conrad J, Kelley RL, Srivastava VJ. 1998. Effect of temperature and pressure on growth and methane utilization by several methanotrophic cultures. Applied Biochemistry and Biotechnology 70:729-738. DOI: 10.1007/BF02920184.

Stolyar S, Van Dien S, Hillesland K, Pinel N, Lie T, Leigh J, Stahl D. 2007. Metabolic modeling of a mutualistic microbial community. Mol Syst Biol 3:93. DOI: 10.1038/msb4100131.

Strong PJ, Xie S, Clarke WP. 2015. Methane as a resource: Can the methanotrophs add value? Environmental Science and Technology 49:4001-4018. DOI: 10.1021/es504242n.

Sugioka K, Nakano M, Naito I, Tero-Kubota S, and Ikegami Y. 1988. Properties of a coenzyme, pyrroloquinoline quinone: generation of an active oxygen species during a reductionoxidation cycle in the presence of NAD(P)H and O2. Biochim Biophys Acta 964:175-182. 10.1016/0304-4165(88)90164-X

Svenning MM, Hestnes AG, Wartiainen I, Stein LY, Klotz MG, Kalyuzhnaya MG, Spang A, Bringel F, Vuilleumier S, Lajus A, Médigue C, Bruce DC, Cheng J-F, Goodwin L, Ivanova N, Han J, Han CS, Hauser L, Held B, Land ML, Lapidus A, Lucas S, Nolan M, Pitluck S, Woyke T. 2011. Genome sequence of the Arctic methanotroph Methylobacter tundripaludum SV96. Journal of bacteriology 193:6418-6419. DOI: 10.1128/JB.05380-11. zklarczyk D, Morris JH, Cook H, Kuhn M, Wyder S, Simonovic M, Santos A, Doncheva NT, Roth A, Bork P, Jensen LJ, and von Mering C. 2017. The STRING database in 2017: quality-controlled protein-protein association networks, made broadly accessible. Nucleic Acids Res 45:D362-D368. 10.1093/nar/gkw937

Temperton B, Giovannoni S. 2012. Metagenomics: microbial diversity through a scratched lens. Current Opinion in Microbiology. Current Opinion in Microbiology 15:605-612. DOI: 10.1016/j.mib.2012.07.001.

Theisen AR, Murrell JC. 2005. Facultative methanotrophs revisited. Journal of bacteriology 187:4303-4305. DOI: 10.1128/JB.187.13.4303-4305.2005.

UniProt Consortium T. 2018. UniProt: the universal protein knowledgebase. Nucleic Acids Res 46:2699. 10.1093/nar/gky092

Vallino JJ. 2003. Modeling Microbial Consortiums as Distributed Metabolic Networks. The Biological Bulletin 204:174-179. DOI: 10.2307/1543554.

van Teeseling MC, Pol A, Harhangi HR, van der Zwart S, Jetten MS, Op den Camp HJ, and van Niftrik L. 2014. Expanding the verrucomicrobial methanotrophic world: description of three novel species of Methylacidimicrobium gen. nov. Appl Environ Microbiol 80:6782-6791. 10.1128/AEM.01838-14

Wartiainen I, Hestnes AG, McDonald IR, and Svenning MM. 2006. Methylobacter tundripaludum sp. nov., a methane-oxidizing bacterium from Arctic wetland soil on the Svalbard islands, Norway (78 degrees N). Int J Syst Evol Microbiol 56:109-113. 10.1099/ijs.0.63728-0

Whalen SC, Reeburgh WS. 1990. Consumption of atmospheric methane by tundra soils. Nature. DOI: $10.1038 / 346160 \mathrm{a} 0$.

Yu Z, Krause SMB, Beck DAC, Chistoserdova L. 2016. A synthetic ecology perspective: How well does behavior of model organisms in the laboratory predict microbial activities in natural habitats? Frontiers in Microbiology. DOI: 10.3389/fmicb.2016.00946.

Yvon-Durocher G, Allen AP, Bastviken D, Conrad R, Gudasz C, St-Pierre A, Thanh-Duc N, Del Giorgio PA. 2014. Methane fluxes show consistent temperature dependence across microbial to ecosystem scales. Nature. DOI: 10.1038/nature13164.

Zengler K. 2009. Central role of the cell in microbial ecology. Microbiol Mol Biol Rev 73:712729. DOI: 10.1128/MMBR.00027-09.

Zengler K, Palsson BO. 2012. A road map for the development of community systems (CoSy)

Peer] reviewing PDF | (2020:02:45973:2:0:NEW 28 May 2020) 
903

906

907

908

909

910

911

912

913

914

915

916

917

918

biology. Nature Reviews Microbiology 10:366-372. DOI: doi.org/10.1038/nrmicro2763.

Zhao S-J, Hanson RS. 1984. Isolate 761M: a New Type I Methanotroph That Possesses a Complete Tricarboxylic Acid Cycle. Applied and Environmental Microbiology 48:1237 LP -1242 .

Zheng Y, Huang J, Zhao F, and Chistoserdova L. 2018. Physiological Effect of XoxG(4) on Lanthanide-Dependent Methanotrophy. Mbio 9. 10.1128/mBio.02430-17

Zhuang K, Izallalen M, Mouser P, Richter H, Risso C, Mahadevan R, Lovley DR. 2011. Genome-scale dynamic modeling of the competition between Rhodoferax and Geobacter in anoxic subsurface environments. ISME J 5:305-316. DOI: 10.1038/ismej.2010.117.

Zomorrodi AR, Islam MM, Maranas CD. 2014. D-OptCom: Dynamic Multi-level and Multiobjective Metabolic Modeling of Microbial Communities. ACS Synthetic Biology. DOI: $10.1021 / \mathrm{sb} 4001307$.

Zomorrodi AR, Maranas CD. 2012. OptCom: A multi-level optimization framework for the metabolic modeling and analysis of microbial communities. PLoS Computational Biology. DOI: 10.1371/journal.pcbi.1002363. 


\section{Table 1 (on next page)}

Model statistics for Methylobacter and Methylomonas 
1 Table 1:

2 Model statistics for Methylobacter and Methylomonas

\begin{tabular}{|c|c|c|}
\hline Models & Methylobacter & Methylomonas \\
\hline Genes & 704 & 658 \\
\hline Reactions & 1404 & 1391 \\
\hline Metabolites & 1329 & 1378 \\
\hline Blocked reactions & 660 & 672 \\
\hline
\end{tabular}

3

4

5 
Table 2 (on next page)

High and limiting nutrient conditions for community simulation. 
1 Table 2: High and limiting nutrient conditions for community simulation

\begin{tabular}{|l|c|c|c|}
\hline \multicolumn{1}{|c|}{ Condition } & $\begin{array}{c}\text { Methane uptake } \\
\text { (mmol/gDCW.hr) }\end{array}$ & $\begin{array}{c}\text { Oxygen uptake } \\
\text { (mmol/gDCW.hr) }\end{array}$ & $\begin{array}{c}\text { Nitrogen uptake } \\
\text { (mmol/gDCW.hr) }\end{array}$ \\
\hline A (high C, high O, high N) & 100 & 100 & 100 \\
\hline B (high C, limiting O, high N) & 100 & 50 & 100 \\
\hline C (high C, high O, limiting N) & 100 & 100 & 40 \\
\hline D (limiting C, high O, high N) & 20 & 100 & 100 \\
\hline
\end{tabular}

2 
Figure 1

Community dynamics showing the fluxes of key shared metabolites and biomass in the community model.

A) High nutrient condition; B) Oxygen limited condition; C) Nitrogen limited condition; and D)

Carbon limited condition

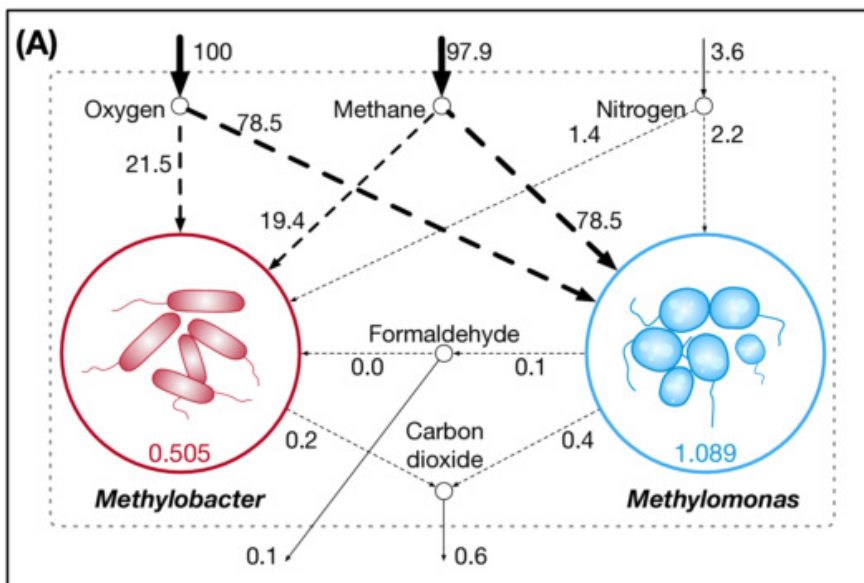

(B)

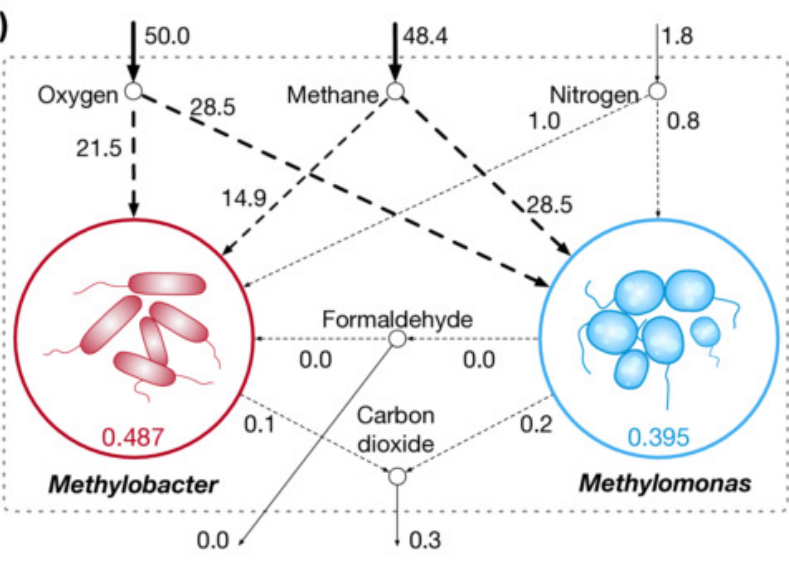

(C)

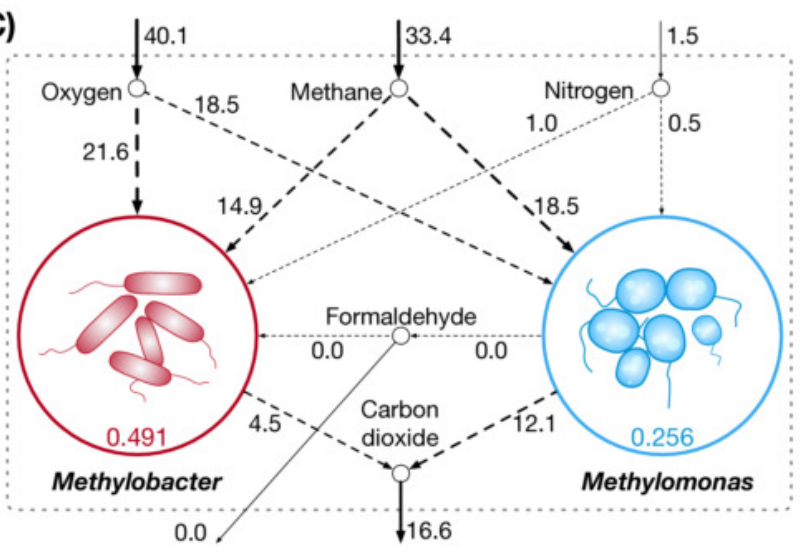

(D)

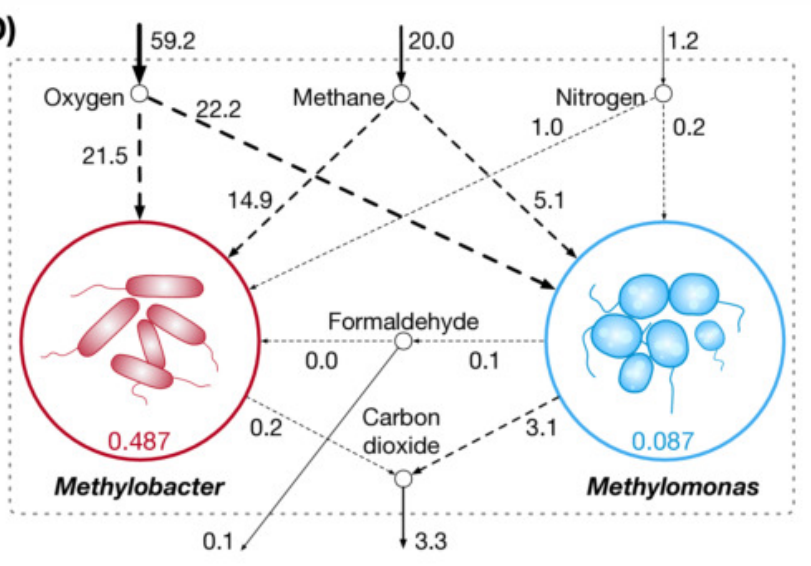


Figure 2

The community composition and total biomass under varying Methane and Oxygen conditions.

The size of each pie chart represents the total community biomass. 


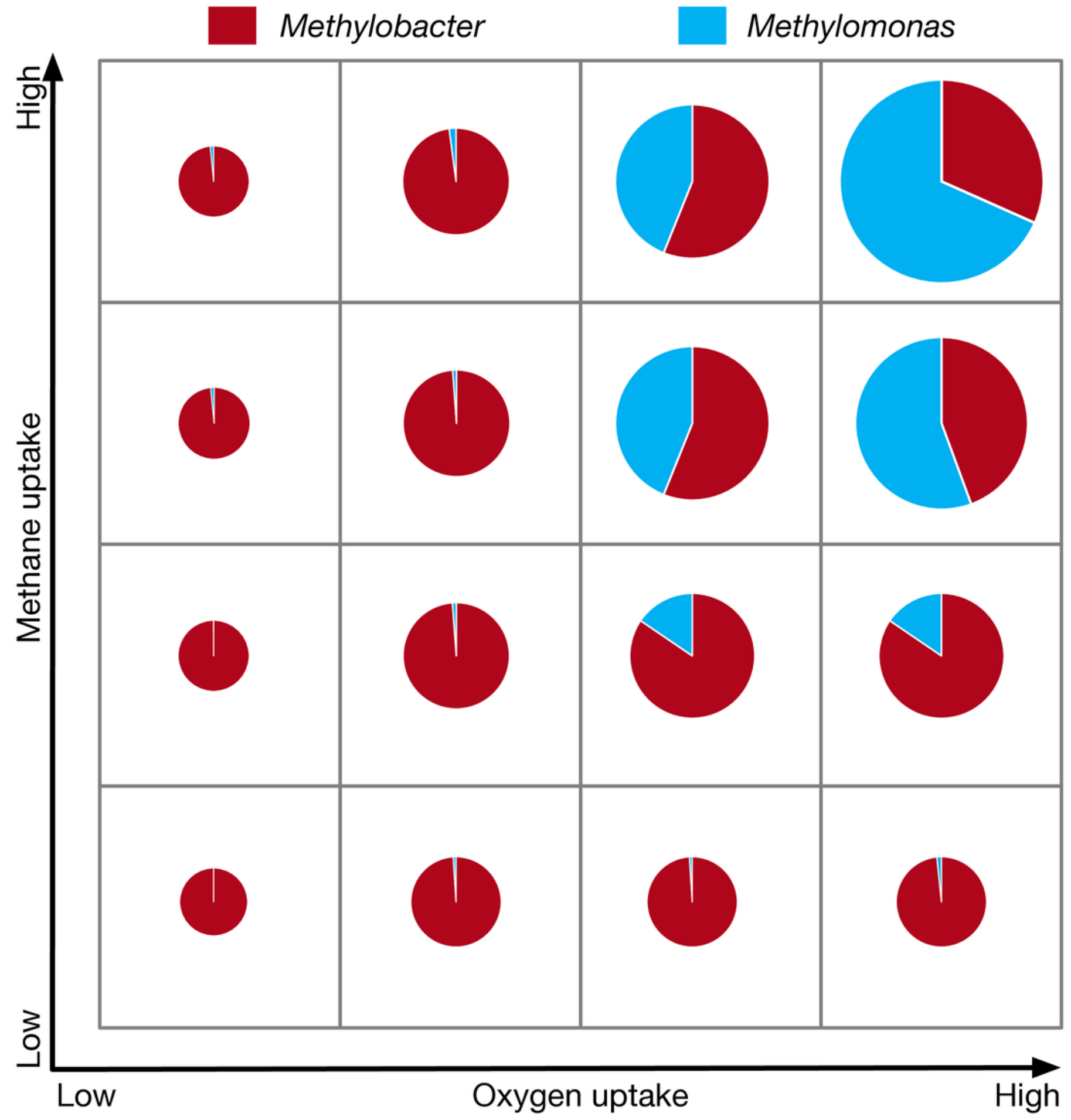


Figure 3

Central carbon metabolism fluxes in Methylobacter (A) and Methylomonas (B) under high nutrient condition.

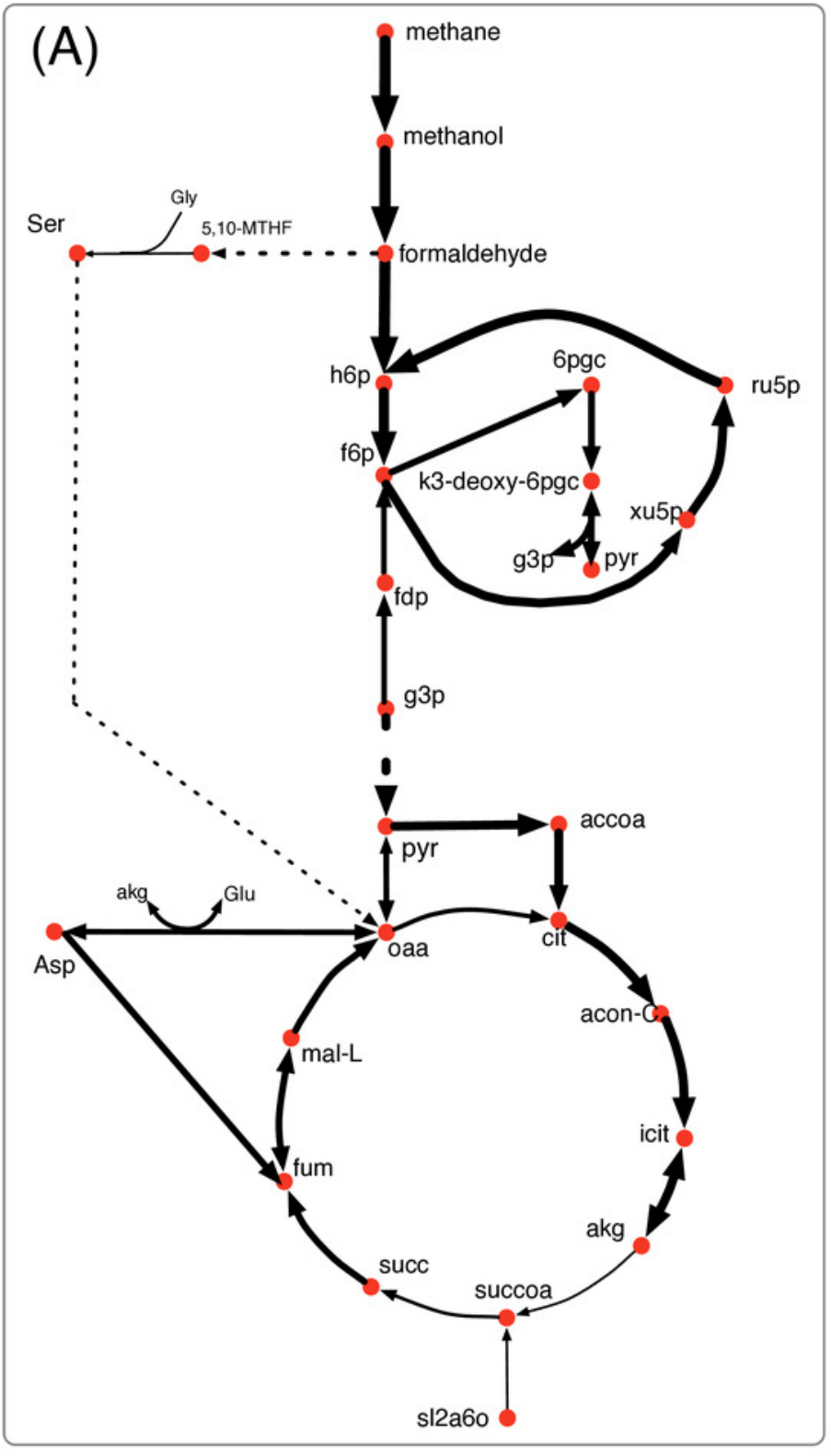

Methylobacter

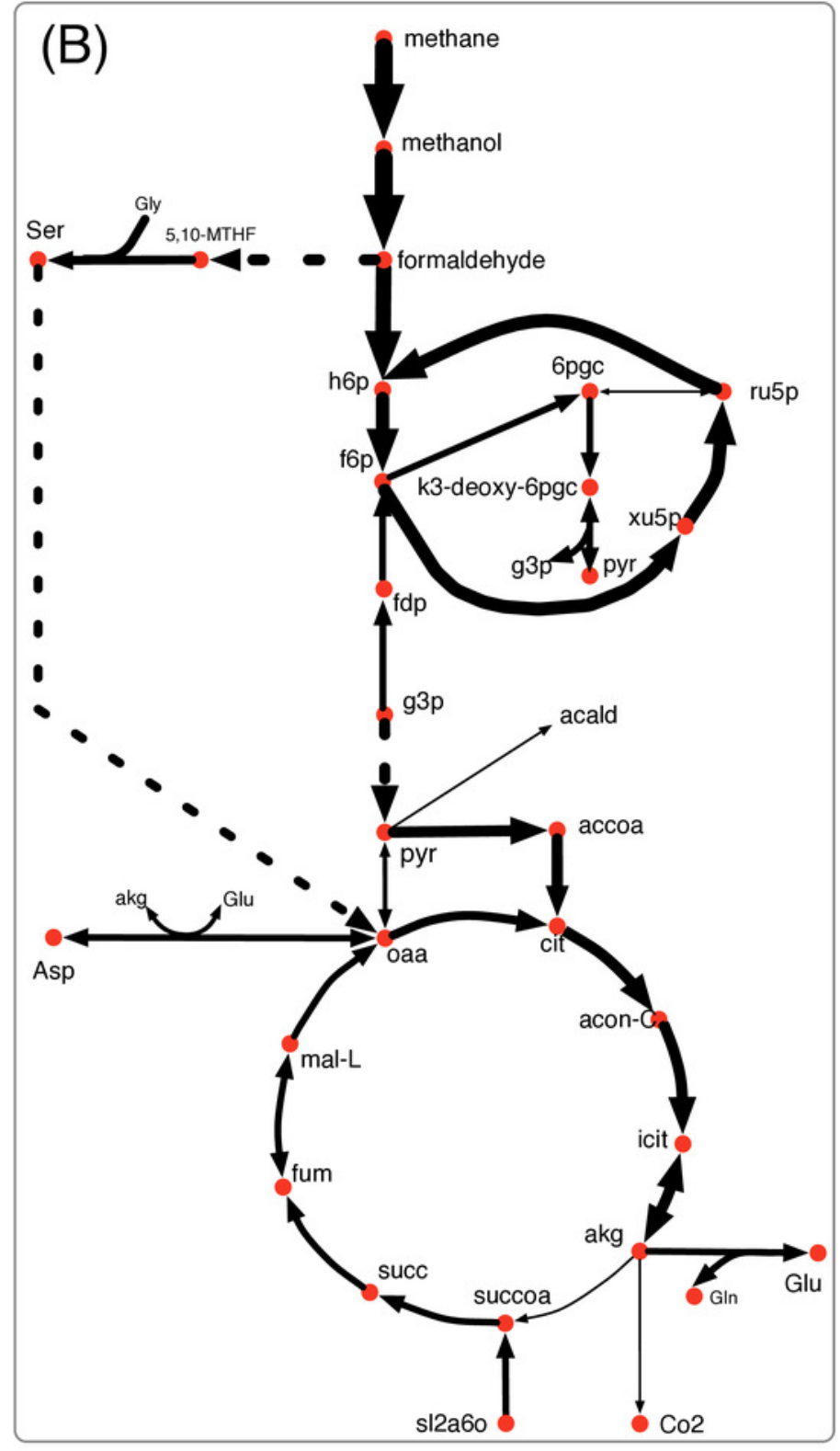

Methylomonas

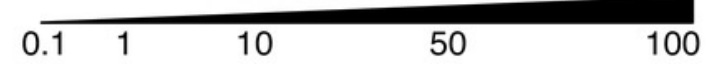


Figure 4

Central carbon metabolism fluxes in Methylobacter (A) and Methylomonas (B) under Oxygen limited condition.

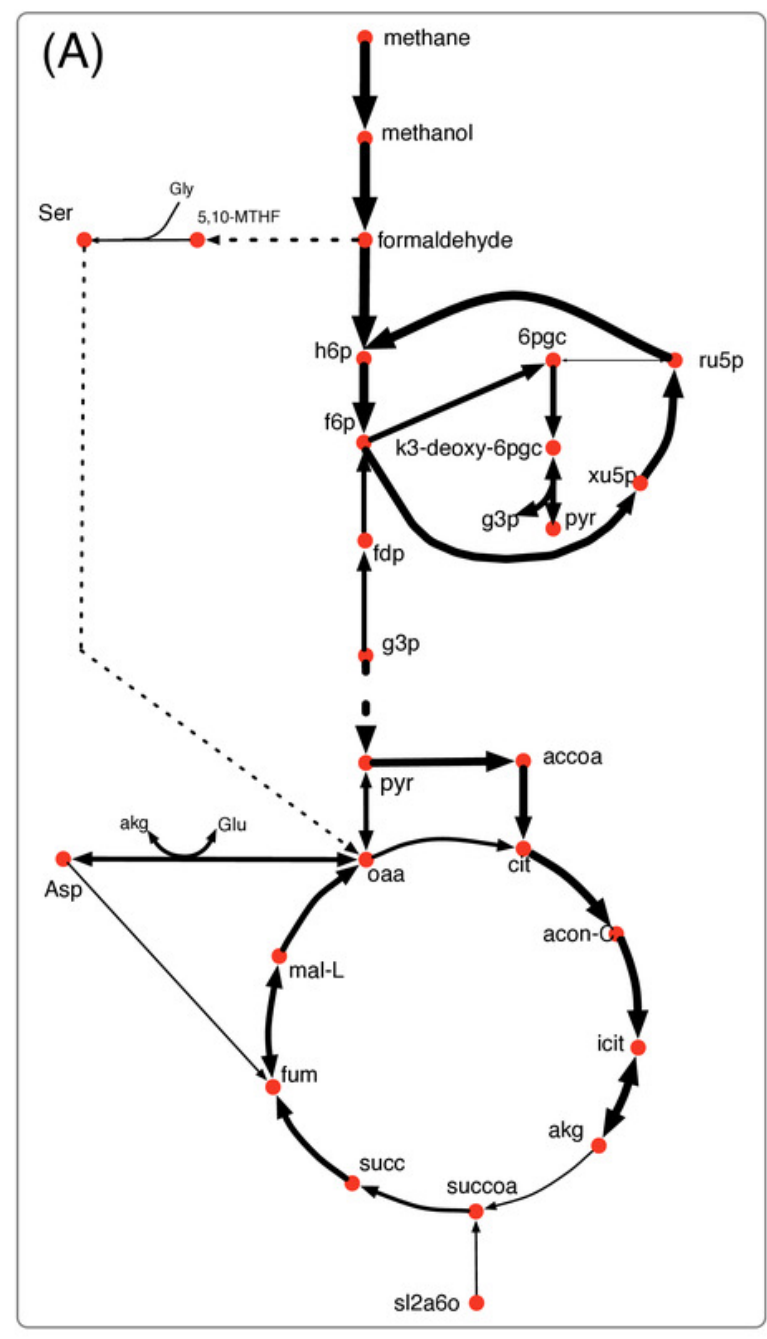

Methylobacter

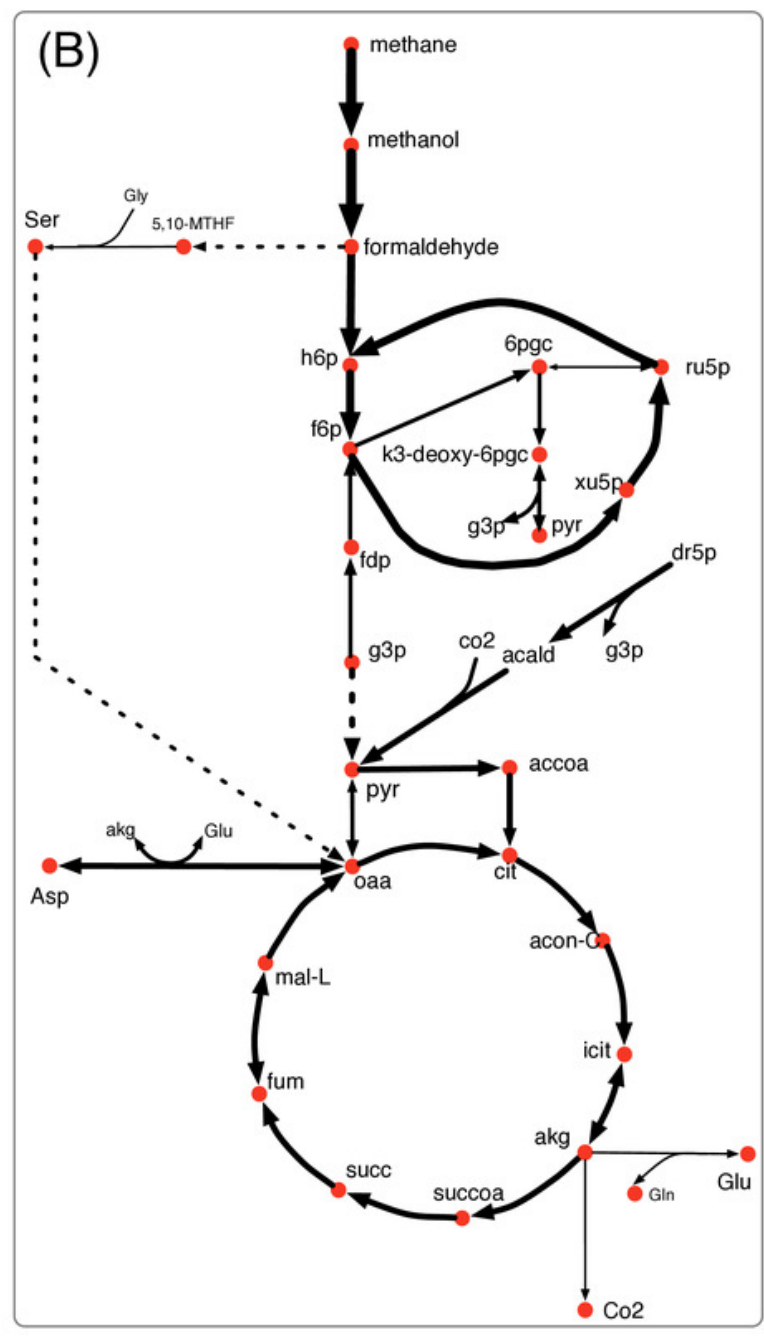

Methylomonas

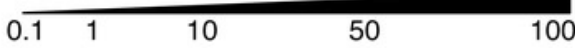


Figure 5

Central carbon metabolism fluxes in Methylobacter (A) and Methylomonas (B) under Nitrogen limited condition.

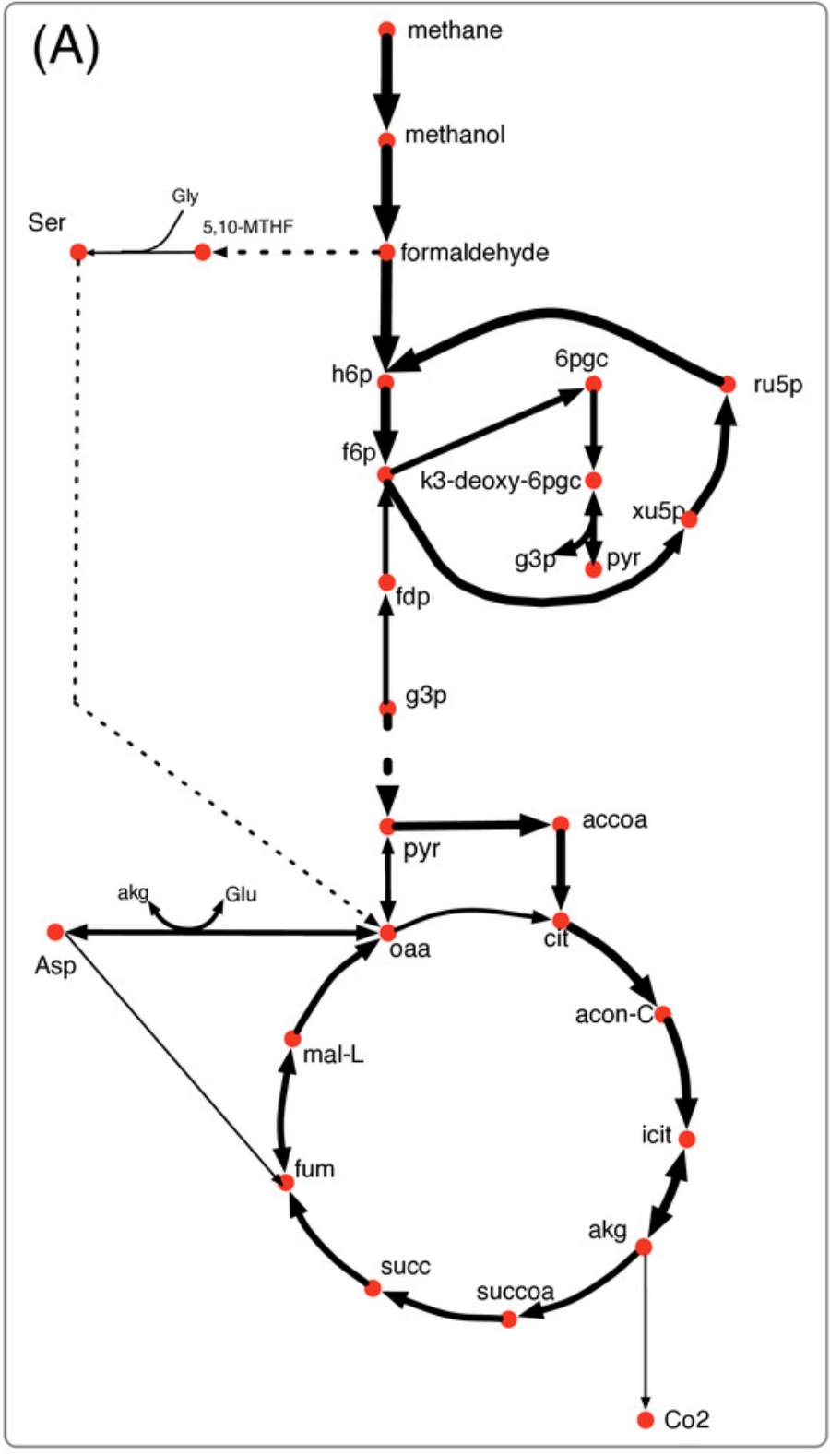

Methylobacter

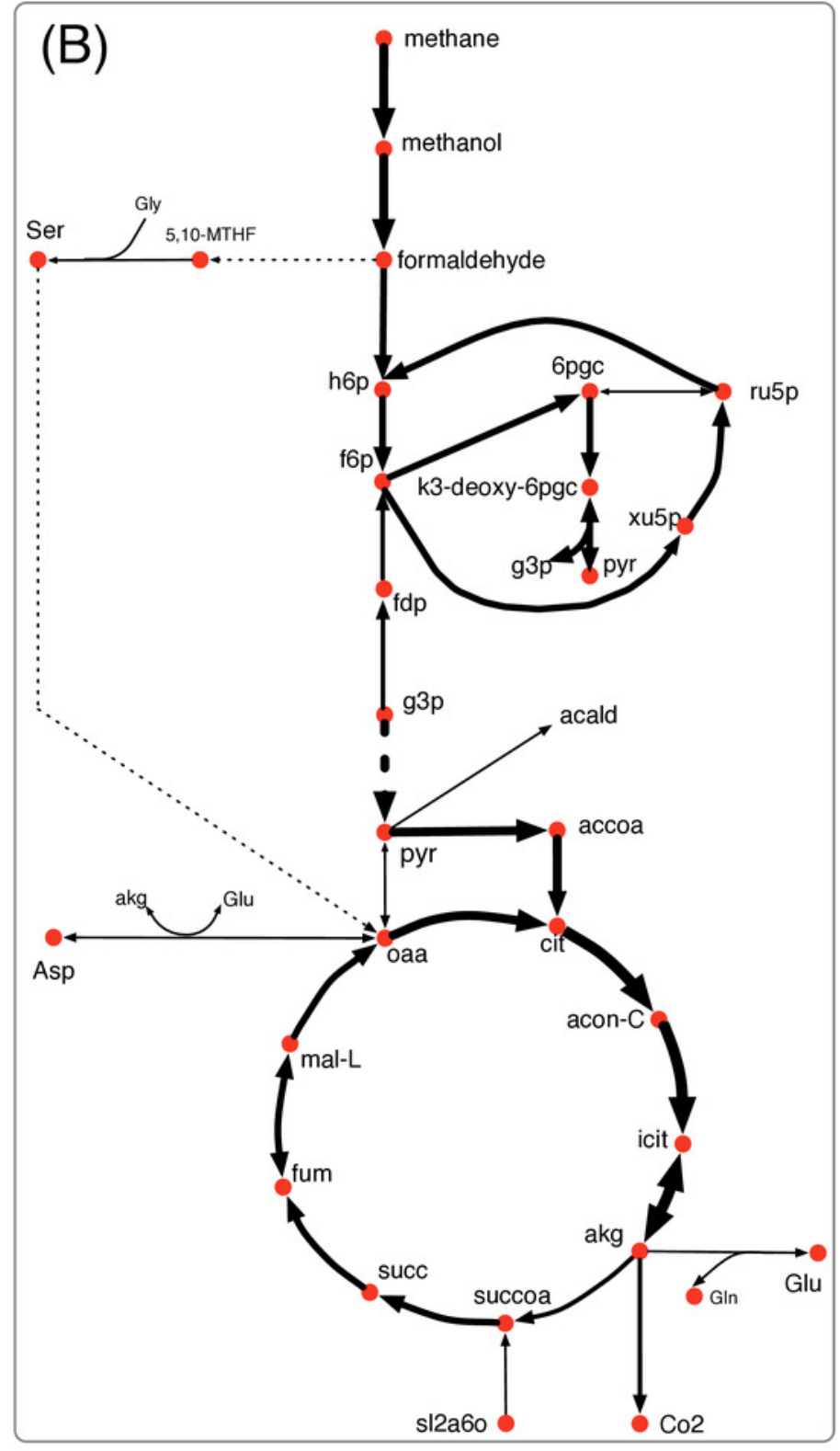

Methylomonas

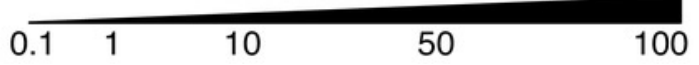


Figure 6

Central carbon metabolism fluxes in Methylobacter (A) and Methylomonas (B) under Carbon limited condition.

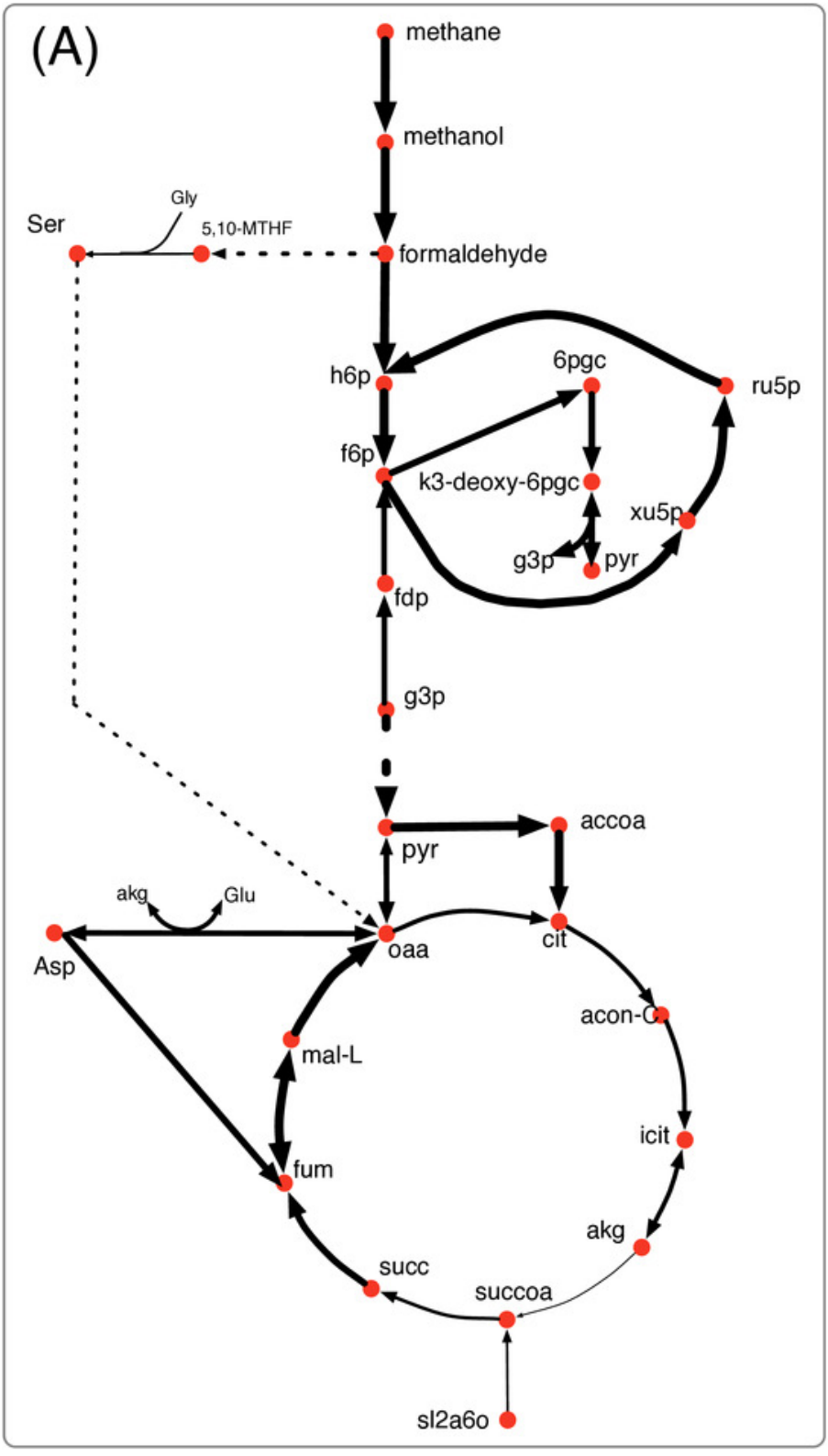

Methylobacter

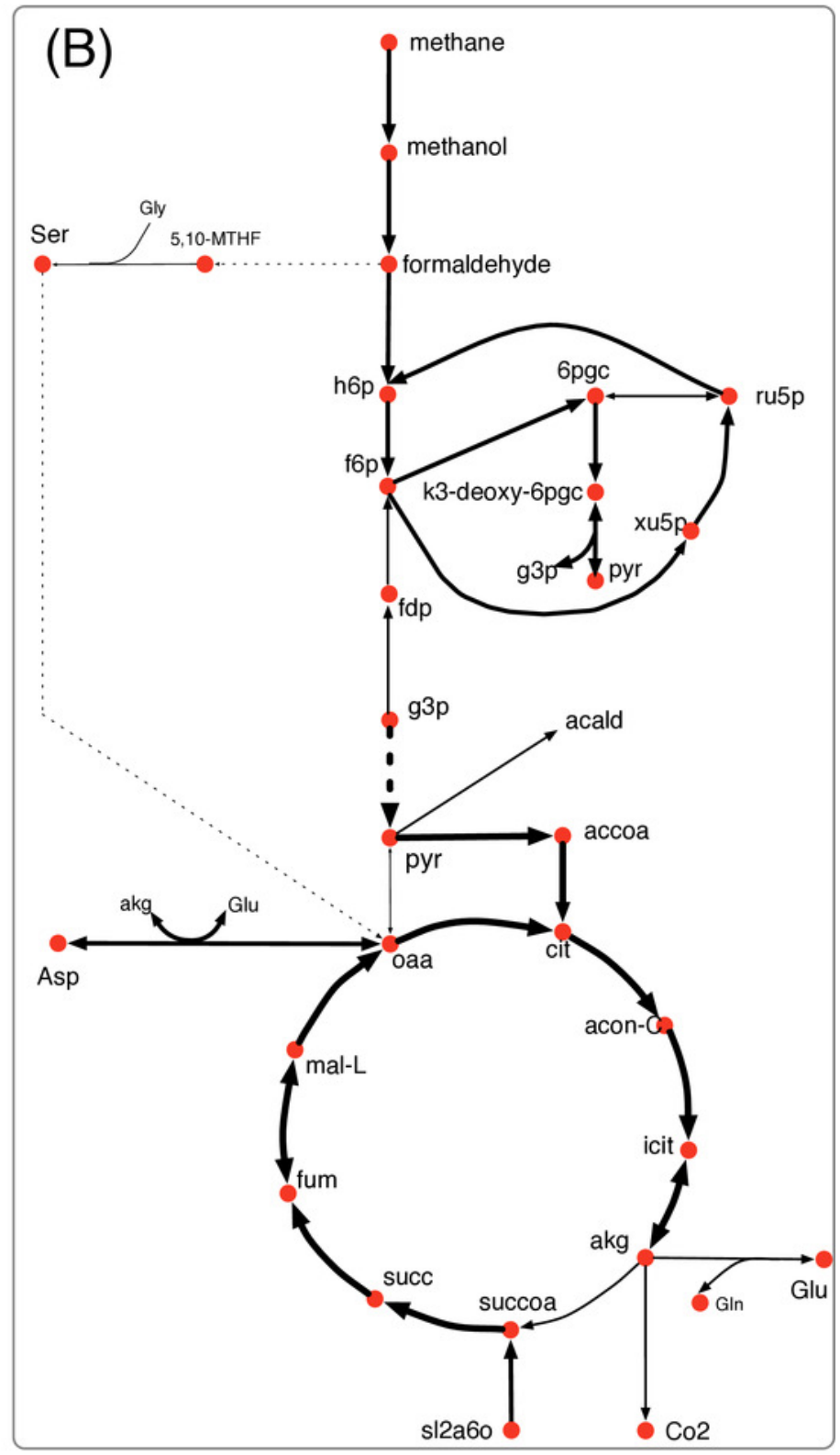

Methylomonas

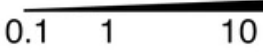


Figure 7

Flux distribution for select metabolites in Methylobacter and Methylomonas under A) Lake Washington sediment-incubated microcosm conditions and B) synthetic co-culture conditions.

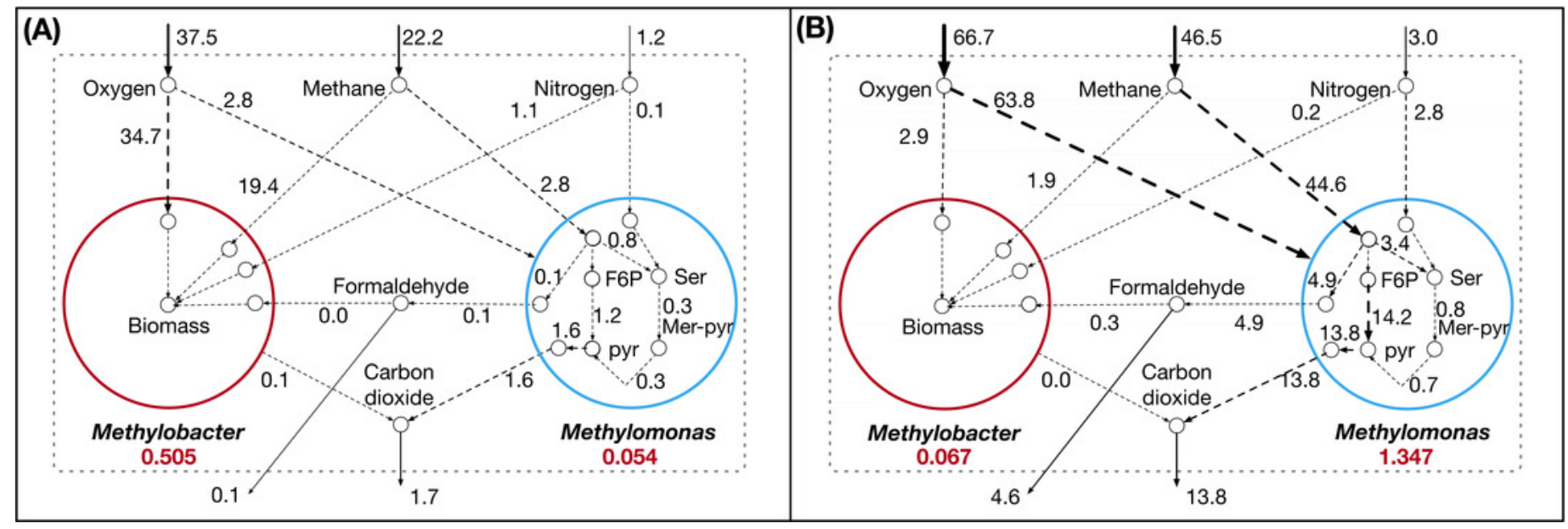

